\title{
Strength Limit of Earth: A New Fundamental Phenomenal Factor for Universe's Precise Scientific Perceptions and Descriptions
}

\author{
Mohammad Salehi Alashti \\ Independent Researcher, Tehran, Iran \\ Email: m.salehi.alashti@hotmail.com
}

How to cite this paper: Alashti, M.S. (2017) Strength Limit of Earth: A New Fundamental Phenomenal Factor for Universe's Precise Scientific Perceptions and Descriptions. Open Access Library Journal, 4: e4034.

https://doi.org/10.4236/oalib.1104034

Received: October 15, 2017

Accepted: October 20, 2017

Published: December 19, 2017

Copyright (@) 2017 by author and Open Access Library Inc.

This work is licensed under the Creative Commons Attribution International License (CC BY 4.0).

http://creativecommons.org/licenses/by/4.0/

(c) (i) Open Access

\begin{abstract}
Intuition of many different unsolved problems and questions remains the "sorest troubles" in basis and applied sciences particular in physics today. Also presented physical models doesn't describe world's framework aright and these make lots of defects to understand sciences and solve scientific problems. Therewith no one can formulate the complicate relation(s) between all existences' and sciences' components. However, no key parameter introduces to discover applicable alphabetic special strings for this purpose till now. No theory exists for help to reconciliation, connection and combination of all correct parts of the current physical framework and theories to construct a whole unification theory for everything through unifying of them. Defining a factor that indicates the earth's strength limit help to reaching mentioned aims and response to unsolved problems in divers sciences such as: How began the universe? How the universe(s) work? Is the speed of light varying imperceptibly? And are the constants of physics changed? Strength limit of earth complicate pivotal and fundamental parameter introduced in this study through different aspects of its specifications and definitions, which helps finding and showing extend connections in addition demonstrates the exact relations of the entire world's components. Results represented that different scientific definitions of this factor which illustrated in this original work show the unity, correlation, continuity and dependency of all universe parts. Moreover, gravity, area density, Euler's constant and earth's strength limit are determinant factors that perform assistant and vital roles in cognition of various sciences' interactions. Depth perception of presented parameter will develop faster scientific discoveries in the world.
\end{abstract}

\section{Subject Areas}

Classical Mechanics, Classical Physics, Geophysics, Theoretical Physics, Multi-Disciplinary Physics, Classical Theories of Gravity, Gravitational Physics, General Physics 


\section{Keywords}

Gravity, Fundamental Forces of Nature, Universe Expansion Rate, Age of the Galaxy, Effective Radius of Earth, Molten Particles, Earth Melting Temperature, Adjusted Earth's Rotation Parameters, Principle Great Structural Revolutionary Mutation Changes Span, Exact Astronomical Unit, The Impact Velocity of Gravity, Exact Precession of the Perihelion of Earth, Accurate Total Number of Seconds in One Year, Earth's Precise Distance from the Sun, Determinant Changes of Particles' Specifications at Different Times, Conversion Factor, Varying Speed of Light

\section{Introduction}

Science is the realization language of the universe. Various aspects of sciences can be cognizing with deep look at all of the universe components and their peculiarities.

Simple observations can result different outcomes in scientific discoveries of the outside and inside also hidden and visible world: wrong, true or a combination of them. In addition, every person may have their self-interpretations from scientific happenstances. Scientists some times during history thought presented theories conduct them to final destination of world's whole realization. But thinkers know would never had the universe and earth whole creation processes during science history while know that have the glimmers and further they are in the beginning way of the identifying unknowns.

Some of the main theoretical and experimental unsolved problems in physics illustrates that existing theories beseem unable to clarify some certain observed phenomenon and experimental outcomes also, there are problems in making experiment(s) to test propounded theories or consider phenomenon in vast useful reliable details [Adopted from [1]].

There is no theory connecting general relativity, relativistic quantum field theory, string theory and standard model as an unsolved problem in physics.

Time contradiction upraise as a problem in combining the frameworks of quantum mechanics with general relativity. Time acts as an independent background in quantum theories as discussed by Sakurai and Napolitano in 2010 [2]. Novello and Bergliaffa in 2003 propound that general relativity behaves with time as a dynamical variable which interacts directly with matter and moreover requires the Hamiltonian constraint to disappear any existence of notion of time's applications as same as quantum theory [Adopted from [3]].

Harmonizing the general theory of relativity that explains gravitation and uses to large-scale structures (stars, galaxies, super clusters) with quantum mechanics that involves with the other three fundamental forces interacting, it is the profound problem in theoretical physics [4].

Proving the unification of gravity as one of the known fundamental forces of nature with the others stands problematical. 
Some physicists deserted classical unified field theories according to classical physics because gravity force's unification with electromagnetism, weak and strong nuclear forces (other fundamental forces of nature) demonstrates difficult [Adopted from [5]].

Describing fundamental interactions through generalized ordinary quantum field theories which include gravity have much serious problems at very high energies which make it is not success as an acceptable effective quantum field theory of gravity. Because the perturbative results are badly divergent and lead to models devoid of predictive power as presented by Hooft in 1974, Veltman in 1975, Goroff \& Sagnotti in 1985, Donoghue in 1995, Weinberg in 1996 and Hamber in 2009 [Adopted from [6]-[11]].

Zee in 2010 proposed that while a quantum theory of gravity probably required according to assimilate general relativity with the quantum mechanics' principles, problems occur when decides to use the common prescriptions of quantum field theory to the gravity force through graviton bosons [Adopted from [12]]. Penrose in 2007 ratiocinated that the problem is the theory has taken up one approach in this way which is not renormalizable and hence cannot be applied to create significant physical predictions. Physicists in this way get most popular radical methods such as string theory and loop quantum gravity because of the quantum gravity problem [Adopted from [13]].

P.A.M. Dirac in 1930 and C.D. Anderson in 1933 explicated that spin is phenomenological and cannot be explained, in non-relativistic quantum mechanics [Adopted from [14] [15]].

Relativistic quantum mechanics is not sufficient for a more precise and whole perfected theory of particle interactions due to the existence of antiparticles [Adopted from [12] [16]]. Messiah in 1981 represented that relativistic quantum mechanics (RQM) does not explain subjects where the number of particles changes; such as in creation and annihilation of matter [Adopted from [17]].

The loop quantum gravity theory is one probable solution for the problem of quantum gravity, as is string theory [Adopted from [1]]. Nicolai, Peeters, and Zamaklar 2005 argued that at this time, no semiclassical limit recovering general relativity has been demonstrate to have actual being. This means that loop quantum gravity's description of spacetime remains unproven at the Planck scale has the right continuum limit which described by general relativity with probable quantum corrections [Adopted from [18]]. Nicolai, Peeters, and Zamaklar in 2005 declared specifically that, the dynamics of the theory is encoded in the Hamiltonian constraint, but there is no Hamiltonian candidate. Other technical problems contain discovery off-shell closure of the algebra limitation and physical inner product vector space, coupling to matter fields of Quantum field theory, fate of the regularization of the graviton in perturbation theory that cause to ultraviolet divergence beyond 2-loops [18].

Goswami, Joshi and Singh et al. in 2006 elucidated that the lack of semi-classical limit leads loop quantum gravity has not yet even caused general relativity created the predictions. As a result of it a problem which plagues all quantum 
gravity's current theories is the absence of experimental observation for that loop quantum gravity makes a prediction not made by the Standard Model or general relativity based on a recent proposal referencing to observation of naked singularities and doubly special relativity as a part of a loop quantum cosmology [Adopted from [1] [19]].

Rovelli in 2000 and Hamber in 2009 explained that in spite of extent efforts, there is no complete and consistent theory of quantum gravity is currently known, even though a number of promising candidates exist [11] [20]. One candidate toward to full theory of quantum gravity is string theory. A quantum theory not of point particles, but of minute one-dimensional extended unified description of all particles and interactions, including gravity, the price to pay is unusual features which described by Green, Schwarz \& Witten in 1987 and Ibanez in 2000 [21] [22].

The findings of the string theory landscape in 2003 by Michael R. Douglas [Adopted from [23] [24]] proposes that string theory has a large number of inequivalent false vacua which expressed by Ashok, and Douglas in 2004 [Adopted from [23] [25]] led to much discussion of what string theory might finally be anticipated to presignify, and how cosmology can be united into the theory which illustrated by Rickles in 2014 [Adopted from [23] [26]].

Nieuwenhuizen in 1981 proposed that supergravity as a field theory which combines the supersymmetry and general relativity's principles can be seen elicits usually from local supersymmetry [Adopted from [27]].

Also superstring theory is according to supersymmetry. But, the lack of any signs and experimental verification of supersymmetry, even in higher energy region, with absence of any supersymmetric particles' discovering with contemplate that no report on suggesting large extra dimensions from LHC, it is unlikely limit the discovery of supersymmetry at CERN which considered by Woit in 2011, Cassel, Ghilencea, Kraml, Lessa, Ross in 2011, Falkowski in 2011, Tapper in 2010 and Jha Alok in 2013 [Adopted from [28] [29] [30] [31] [32]].

Researchers have tried to develop the Standard Model theoretically and experimentally as a complete unified field theory or a theory of everything, which clarifying all physical phenomena including constants. But standard Model has deficiencies. Standard Model's self-stability has not been demonstrated mathematically [Adopted from [33]].

Neutrinos' mass which experiment represent it at CERN in 2010, does not accommodate with classic Standard Model [Adopted from [34]].

Although it accosts strong and electroweak interactions but the Standard Model does not consistently illustrate gravitation, the canonical gravitation theory and general relativity, in terms of quantum field theory. Although would not account physical confirmation of a theoretical particle known as a graviton. One reason is that quantum field theories of gravity usually break down before attaining the Planck scale [Adopted from [33]].

Blumhofer and Hutter in 1997 discussed that the Standard Model needs 19 
numerical unrelated and arbitrary constants values. It seems affirming neutrino mass that will require additional 7 or 8 arbitrary constants parameters [Adopted from [35]].

It is not probable to make a consistent quantum field theory including elementary scalar particles. The Standard Model has contradictions with Standard Model of cosmology. There is no description for the apperceived cold dark matter's amount and its contributions to dark energy as a common repugnance. In addition it is difficult to reconcile the matter and antimatter asymmetry. The convergence and homogeneity of the apparent universe over large distances beseems to need another mechanism which would also establish the development of the Standard Model. Although the expanded Standard Model, can explain why neutrinos have masses, but the characteristics of neutrino's mass are still unclear. Suggested Theory of Everything has not been widely encountered, used, recognized and substantiated. As a result, currently no reliable theory exists for all aspects of the very early universe [Adopted from [33]].

Modern endeavors to formulate $\mathrm{M}$-theory are according to matrix theory typicality but a complete mathematical formulation structure of M-theory which provides a framework for extending a unified theory of all the fundamental forces of nature is not known [Adopted from [36]].

Composite Higgs models is the contemplative developments of the Standard Model in particle physics where the Higgs boson have a bound state of new strong interactions for the creation reason of complication to express the various energy scales which emerge in the particle physics' fundamental interactions. But nonetheless, there are no direct and indirect detected signs about that the Higgs and other Standard Model particles are composite from 2015 as yet that expounded by Giudice in 2013 [Adopted from [37] [38]].

Various reasons indicate that the current theoretical physical framework is incomplete because all experiments and observations does not definitely needs to them for their explanation. However that one needs a whole theoretical gravity and a theory for all things for their completion which unified all interactions with an independence background also provide an extendable practical model of particles physics that set to the real experiments by contemplate all dimensions, viable alternatives and all properties and time role in the entire existence.

Likewise, current physical models cannot find and present all responds of science's questions specially about is there any existence and how the worlds coming into existence and what is its exact conditions, physicogenesis, biogenesis and structures of the creations plus other sciential genesis (or sciento-genesis) too.

Moreover, there are not a theory and even quintessential factor that illustrates all fundamental physical constants' values. Also, therewith the quantum field theory the research is still continues for a more universal theory of matter and energy. While this new concept which presented in this study can use to connect true parts of various proposed physical frameworks such as general relativity and the relativistic quantum field theory with new discovered models and results, ef- 
fectively as a toe and theory of everything (ToE), and precisely clarify how exactly tries to improve it.

Discern the knowledge behind the true transpicuous story of the creation respond to many important questions: What things imply to universe(s) exactly?; Why universe should be existed scientifically?; How the origin of the universe created?; How the universe exist(ed) and continue to existing?; How were the beginning of the universe?; How does the universe evolve?; How are the exact framework of the universe?; What is the story of creation behind science and realizing the knowledge?; How the world changes?; What are the forces that impact and govern in these changing?; Are the forces word true for realizing the cosmic evolution?; How the universe existed in to un-existed world?; How universe formed and be in existed initial ancient world?; How the earth and other creatures created in to existed world?; What happened from a split second after earth initial creation into existence (which still don't know what that these exactly until today after existence and evolvement processes)?; What is the source of gravity?; What are the origins of gravity?; How the origins of the gravity created? ...

The previous knowledge presented that Newton's laws combined with universal gravitation and classical electrodynamics are not adequate to use in noticeable status such at very small scales, very high speeds (when the involved speeds are much faster than the speed of light) and very strong gravitational fields. While said that, the mentioned laws cannot be applied to explain phenomena like as conduction of electricity in a semiconductor, optical properties of substances, errors in non-relativistically corrected GPS systems and superconductivity [Adopted from [39]]. Also Jacob Lubliner in 2008 exposed to discussion that they are inappropriate to characterize the motion of rigid bodies and deformable bodies [Adopted from [40]]. Accordingly elucidation and description of these phenomena needs more sophisticated physical theories which these manners are an apparent analysis. But profoundly, Newtonian gravity is useful to explain a vast range of physical sciences phenomena by making a unified measurable interpretations via precise views in the deep knowledge concealed behind their sciences through a complicated substantial strength limit parameter which having acquired and refinement worldly knowledge in parallel interaction with gravity, area density, Napier's number and other fundamental factors.

Accordingly to above illustrations as the main purpose of the present researches work must ask this question: Is there any primordial factor with leading key roles to solve mentioned aims in the future of scientific researches? Strength limit is the key parameter for adjusting, joining and combining various scientific ideas and theories. This leads to comprehend all procedures of final creation of all existing things, atoms, stars, galaxies and others then making the complete continuum scientific model of the structures of the universe specially physical model. Strength limit uncover and shaped a unified pervasive theory of all interactions specially particles interactions.

Response to interdisciplinary fundamental key questions accurately also is an important basis work. Strength limit factor of earth which included gravity and 
other fundamental factors perform procreator and impressive roles for discovery of mathematical frame work of world in a unity model, extended precision and complicates relations between various scientific features of universe, interaction of particles, combination and equivalency of all forces and algorithm and structure of gravity with other known and un-known forces. In this study for the first time, various definitions of earth's strength limit parameter that seems simple but a new complicate parameter, introduced and recommended for help in response to lots of subjects in various sciences such as:

Determining accurate age of the earth and other planets; Computing accurate age of the galaxy; Characterizing accurate age of the universe; Showing the variations of earth's specifications; Adjusting of calculated features of earth; Cosmic speed measurement; Specifying earth's rotation peculiarities; Ascertain the accurate light velocity; Distinguish the variations of light velocity [Is speed of light variable (special in various epochs)?]; Adjusting speed of light and distance that light travel during one year precisely; Assessing endurable temperature by earth; Knowing of ecosystem changes in earth; Finding out specifications of great climate, continental and structural changes of planets like earth; Discovering determinant and basis particles; Showing changes in specifications of particles in different time; Adjusting scientific units; recognizing exact distance between planets such as the precise distance of earth from other planets; Determine the velocity of smallest particles; Revealing gases changes in universe, galaxies and planets; Accurate studying and analyzing of gases changes in air; Measuring gases changes in atmosphere (determining reduce and increase values of gases in atmosphere) and it's impacts on various phenomenon such as biological events; Precise measurements of biological specifications of human beings; Effective radius of earth determination in global continuum gravitational field; Finding perihelion point and distance, Discovery of un-known fundamental forces in universe; Defining existence as a set of universe and every things in it; Description of main keys in communications between different sciences; Description of errors in physics theories; Solving the greatest unsolved problems in science such as unsolved problems in physics...

Even so new definitions of Euler's constant and area density of earth are presented based on various features according to strength limit factor. New simple and complicate philosophical approaches and theories defined and used based on mathematical philosophy of strength limit number for help to realizing various aspects of this study and universe scientific and philosophical cognition. This article written based on simplified mathematical structure.

This theoretical regular research study with scrutiny approaches introduces Strength Limit of Earth as an overture for help to achieve mentioned objectives in the future. In this scientific paper, Section 2 illustrates the theory for the suggested fundamental factor. Section 2.1 represents a brief description of strength limit of earth. Section 3 explains the characterization of strength limit number of earth. Section 4 demonstrates various definitions of strength limit factor of earth based on scrutinizing previous presented explanations and new definitions in 
accordance to thickness of homosphere, distance that light travel during one year, effective radius of earth, molten particle specifications, age of the galaxy, number of cells in human body, also from different approaches. Section 5 clarifies results and some applications of strength limit of earth. Section 6 defining perceptible concepts based on all mentioned results in this research. Section 7 affirms comprehensible general and philosophical approaches according to this study. Section 8 expresses summary, discussion and conclusion about the research study. Finally Section 9 implies some of the significances of this study for future of scientific researches.

\section{Theory}

\subsection{Brief Description of Strength Limit of Earth}

When materials' cohesion changed with different reasons like structural un-stabilities, tectonic actions, geological structures, engineering geology problems and etc., then cohesion values between particles that named strength limit factor (in general form shows with $\boldsymbol{X}_{c \text {-Earth }}$ abbreviation) are less than about 65 percent (exactly equal to $64.712157172243419172410449319661 \%$ ) of own first value, therewith instability changes begun. In this condition micro cracks create also will develop and finally different results of instabilities such as failure happen. This parameter is constant instantly in a defined period of time (or throughout the duration of an structural interval) pending life time of earth which called cohesion constant limit number $\left(\boldsymbol{X}_{\boldsymbol{C} \text {-Constant }}\right.$ or $\left.\boldsymbol{X}_{\text {CCLN }}\right)$ and on the other hand is variable that named cohesion variable limit number ( $\boldsymbol{X}_{\text {C-variable }}$ or $\boldsymbol{X}_{\mathrm{CVLN}}$ ). Strength limit of earth showed and portend that what values remain active from $1 \mathrm{MPa}$ or 1 percent of any strength or from 100 percent of total strength forces. Instabilities of structures like earth (and etc.) begun when their strength limit parameter decreases continuously and reach to $\left[X_{c-\text { Earth }} \times 100\right] \%,\left[\left(\frac{\% X_{c-\text { Earth }}+\% \text { G.S.Ns }}{2}\right) \times 100\right] \%$ and [(Golden section numbers) $\times 100] \%$ of theirs first own value. Then approach to $50 \%$ of preliminary amount and in critical stages strength limit parameter pass from $[(1-$ G.S.Ns $) \times 100] \%$ and $\left[\left(1-\frac{\% X_{c-\text { Earth }}+\% \text { G.S.Ns }}{2}\right) \times 100\right] \%$ of its primary value and ruptures begin in structures. The Golden section number is 0.6180339887. Therefore complete decomposing times and procedures arise during and after that strength limit parameter reduce yet and its value passes from $\left[\left(\mathbf{1}-\boldsymbol{X}_{\boldsymbol{c}-\text { Earth }}\right) \times \mathbf{1 0 0}\right] \%$ of preliminary value [Adopted from [41]].

Note: Present phrase in this article for various features of earth and universe means the new discovered value for strength limit, gravity and other features of earth and cosmos. This subject shows their changes during times. Old values defined according to 4.5501750498945017745329167415032 billion years age of the earth from its complete formation time. And present values calculated based on 4.7303652362904 billion years age of the earth which starts from its initial creating time. 


\section{Characterization of Strength Limit Number of Earth; ( $\mathrm{X}_{\mathrm{C} \text {-Earth) }}$}

Earth's strength limit parameter propounds solutions for various complicate unsolved scientific questions in many fields. This basis structure leads to showing the complicate and simple scientific relevancies between all constituent parts of the entire universe like as many forces that acting together and having the same effects or un-same kinds. This limit feature invisible equilibrium between all forces which adjoin particles in the universe.

\section{Calculations of Earth's Strength Limit}

\subsection{Previous Researches: Brief Previous Presented Definitions of Strength Limit of Earth}

Some definitions of strength limit of earth based on paper entitled "Accurate age of the earth calculation by a new fundamental parameter" which published in February 2015 presented in parts $4.1 .1,4.1 .2$ and 4.1 .3 briefly as:

\subsubsection{Independent Determination of Strength Limit by Standard Gravity and Area Density for Earth}

Gravity is a tensional regularizer forces that equalize state of materials in the universe. Strength limit factor of earth is the strength state of universe overall stability field due to balanced forces such as gravity and plays significant roles in the structural resistance of gravitational fields. Strength limit of earth demonstrates the equilibrium limit between resisting and disturbing forces in universe gravitational field and besides in equivalency field of the entire world (universe overall stability field). Earth's strength limit number in general, old and present forms are given by below equations through standard gravity and Euler's constant [Adopted from [41]]:

$$
\begin{aligned}
X_{C-\text { Constant }} & =X_{C \text {-earth }}=\text { Gravity } \times \text { Area density }=g_{0} \times \text { A.D }=g_{0} \times \frac{1}{e^{e}} \\
X_{C-\text { Earth,Old }} & =g_{0-\text { Old }} \times \text { A.D } \\
& =0.64712157172243419172410449319661(\text { MPa or \%) } \\
X_{C-\text { Earth,Now }} & =g_{0-\text { Now }} \times \text { A.D } \\
& =0.6727480488031732255208148942682(\text { MPa or \%) }
\end{aligned}
$$

where [41] $\boldsymbol{X}_{\text {C-earth }}$ is the strength limit number of earth or is one of the M.S.A.'s fundamental numbers, $\boldsymbol{g}_{0}$ is the standard gravitational acceleration or standard acceleration due to gravity or earth's standard surface gravity that's equal to $9.80665 \mathrm{~m} / \mathrm{s}^{2}$ [42], $\boldsymbol{X}_{C \text {-Earth,Old }}$ is the cohesion constant limit number of earth or strength limit parameter of earth or strength of existence global gravitation according to old standard gravity and equal to $0.64712157172243419172410449319661 \mathrm{MPa}, \boldsymbol{X}_{C-\text { Earth,Now }}$ is the cohesion constant limit number of earth or strength limit parameter of earth or strength of existence global gravitation according to present standard gravity and equal to $0.6727480488031732255208148942682 \mathrm{MPa}, \boldsymbol{g}_{0-\text { Old }}$ is the old gravity according 
to old strength limit of earth, $\boldsymbol{g}_{\mathbf{0}-\text { Now }}$ is the present gravity according to present strength limit of earth, $\boldsymbol{e}$ is the simply Euler's constant or Napier's constant, $\boldsymbol{e}^{\boldsymbol{e}}$ is equal to $15.15426224147926418976043027263 \mathrm{~m}^{2} / \mathrm{Ggr}$ which means every value of gravitation is effect on $\boldsymbol{e}^{e}$ square meter of every parts of earth or every things near the earth with 1 Ggr mass. In-fact $\boldsymbol{e}^{e}$ is the impact surface area of gravity on 1 Ggr mass, $\frac{1}{\boldsymbol{e}^{\boldsymbol{e}}}$ is area density (A.D or $\boldsymbol{D}_{A}$ ) or surface density (S.D or $\left.\boldsymbol{D}_{\boldsymbol{S}}\right)$ and equal to $0.06598803584531253707679018759685 \mathrm{Ggr} / \mathrm{m}^{2}$ or 65.98803584531253707679018759685 ton $/ \mathrm{m}^{2}$ or in other words, $\frac{\mathbf{1}}{\boldsymbol{e}^{\boldsymbol{e}}}$ is the gravitational impact coefficient (g.I.C) or gravitational exponential coefficient (g.E.C) or exponential coefficient of gravitation $\left(\boldsymbol{E} \boldsymbol{C}_{\boldsymbol{g}}\right.$ ) that means every value of gravitation is effects on 0.06598803584531253707679018759685 Ggr in 1 square meter of every parts of earth or every things near the earth. In fact $\frac{1}{e^{e}}$ is the impact coefficient of gravitation on earth's strength factor $\left(\boldsymbol{I} \boldsymbol{C}_{\boldsymbol{g}}\right)$ or earth's component strength factor. This factor is the gravitation's equivalency density $\left(\boldsymbol{g}_{\text {eq.d }}\right.$ ) or equivalency density of gravitation $\left(\boldsymbol{E} \boldsymbol{q} \boldsymbol{D}_{\boldsymbol{g}}\right)$ and on the other hand this is gravitational exponential impact coefficient (g.E.I.C) or gravitation's exponential impact coefficient $\left(\boldsymbol{g}_{\text {eic }}\right)$ [41].

It's required to explain that all general formulas in this article are true based on old and present values of standard gravity and strength limit of earth.

Standard gravity based on Equation (2) derived from below relation in a detailed general form as [Adopted from [41]]:

$$
\begin{aligned}
g_{0} & =\frac{\text { Strength Limit of Earth }}{\text { Area Density }}=X_{C \text {-earth }} \times e^{e}=X_{C \text {-earth }} \times \sqrt{e^{e}} \times \sqrt{e^{e}} \\
& =\frac{X_{C-\text { earth }}}{\frac{1}{e^{e}}}=\frac{X_{C \text {-earth }}}{\frac{1}{\sqrt{e^{e}} \times \sqrt{e^{e}}}}
\end{aligned}
$$

So that old and present gravity of earth based on Equation (4) calculated as:

$$
\begin{aligned}
g_{0-\text { Old }} & =e^{e} * X_{C-\text { Earth,Old }}=9.8066499999999999999999999999999 \mathrm{~m} / \mathrm{s}^{2} \\
& \cong 9.80665 \mathrm{~m} / \mathrm{s}^{2} \\
g_{0-\text { Now }} & =e^{e} * X_{C-\text { Earth,Now }}=10.195000354006777301014825930477 \mathrm{~m} / \mathrm{s}^{2} \\
& \cong 10.2 \mathrm{~m} / \mathrm{s}^{2}
\end{aligned}
$$

Area density is 65.98803584531253707679018759685 ton in one square meter and in fact is equal to 1000 ton (1 Ggr) in 15.15426224147926418976043027263 square meter $\left(\mathrm{m}^{2}\right)$. Therefore area density is determined by [Adopted from [41]]:

$$
\begin{aligned}
\text { Area Density } & =\text { Surface Density }=\frac{1}{e^{e}}=\frac{1}{\sqrt{e^{e}} \times \sqrt{e^{e}}}=\frac{1}{\left(\sqrt{e^{e}}\right)^{2}} \\
& =\frac{X_{C \text {-earth }}}{g_{0}}=\frac{\text { Strength Limit of Earth }}{\text { Standard Gravity }}
\end{aligned}
$$


Area density states clearly the impact area of gravity forces cells (impact components cells) in continuous entire world. This displays the impact components cells of gravitational waves and components cells of impact area. Surface density computed from characteristics of gravitational impact surface and gravitational particles [Adopted from [41]]. Therewith, surface density is such that:

$$
\begin{aligned}
\text { Surface Density } & =\mathrm{S} \cdot \mathrm{D}=D_{S}=\mathrm{A} \cdot \mathrm{D}=D_{A}=\frac{\text { Mass }}{\text { Surface Area }}=\frac{\text { Unit Mass }}{\text { Unit Surface Area }} \\
& =\frac{\text { Mass Scale Unit }}{\text { Surface Area Scale Unit }}=\frac{M}{x^{2}}
\end{aligned}
$$

where [41] A.D or $\boldsymbol{D}_{\boldsymbol{A}}$ or S.D or $\boldsymbol{D}_{\boldsymbol{S}}$ is area density or surface density. A.D is a local surface density or impact surface density. On the other word is surface density for 1 Ggr unit mass scale and in fact is the area density of gravitational particles according to impact surface area, $\boldsymbol{M}$ is Unit mass of gravitational particles and equal to $1 \mathrm{Ggr}=1 \mathrm{Giga}$ gram or $\mathrm{M}$ is Unit mass of gravitational particles impact components cells, $\boldsymbol{x}^{2}$ is Impact surface area of gravitational particles according to area scale unit. It's very important that know this factor is the staple impact surface area of gravitational waves in presence of gravitational forces with contemplate of gravitational particle(s) properties by surface area scale unit and mass scale unit and $x$ is local relocation value of gravitational particles in impact surface during their life time (or during life time of process). $\boldsymbol{X}$ is Unit length or longitudinal stir of gravitational particles during their life time in impact surface limit or longitudinal limit of gravitational particles in their components impact surface during movement's life time. Thereto, $\boldsymbol{X}$ is longitudinal and latitudinal stir of the impact components cells of gravitational waves (even during life time of gravitational particles) or longitudinal and latitudinal stir of components cells of impact area and equal to

$$
\sqrt{\boldsymbol{e}^{e}}=3.8928475749095628043890533507828 \mathrm{Gm} \text {. }
$$

Gravitational particle(s) cause to equivalency in impact world also originates from equivalency state. Unit length, time and force values of gravitational particles are written directly by [Adopted from [41]]:

$$
\begin{aligned}
& X=\sqrt{\frac{M}{\text { Area Density }}}=\frac{F t^{2}}{M}=\sqrt{e^{e}} \\
& =3.8928475749095628043890533507828 \mathrm{Gm} \\
& t^{2}=\frac{M X}{F}=\sqrt{e^{e}}=3.8928475749095628043890533507828 \mathrm{~s}^{2} \\
& t=\frac{M V}{F}=\sqrt{\frac{M X}{F}}=\sqrt[4]{e^{e}}=1.9730300491653853177990045521702 \text { second } \\
& V=\sqrt[4]{e^{e}}=1.9730300491653853177990045521702 \mathrm{Gm} / \mathrm{s} \\
& F=\frac{M V}{t}=\frac{M X}{t^{2}}=\frac{1 \times \sqrt{e^{e}}}{\sqrt{e^{e}}}=1 \mathrm{GN}
\end{aligned}
$$

On the other word $t$ is a component or frustum recycling time of gravitational particles (components recycle or component recycles). 
where [41] $\boldsymbol{t}=$ Unit time $=$ recycle time of gravitational particles $=$ recycle time of gravitational particles in component impact area of gravity forces cells, $F$ = Unit force $=$ Unit force in gravitational particle's process according to unit mass, time and velocity scale $=$ Local force value $=$ Impresser force $=$ Total Impressive force $=$ Impressive force on every particle $=$ Total Impressive forces involve in life time of gravitational particles (life time of gravitational particle's process) $=$ Effecting force by gravitational particles on area $=$ Effecting forces on gravitational particles by area $=1$ newton force in a large surrounding area $=$ Total Involving forces in life time of gravitational particles (life time of gravitational particle's process) and $\boldsymbol{V}=$ Unit velocity of gravitational particle's process according to unit mass, time and force scales.

$\boldsymbol{x}$ is equal to 3.8928475749095628043890533507828 Giga meter and unit velocity is equal to $1.9730300491653853177990045521702 \mathrm{Gm} / \mathrm{s}$ in big scale impact area (non-component impact area) [Adopted from [41]].

The most frequently asked question is what is the impact velocity of gravity in the gravitational interactions field(s)? In fact unit velocity of gravitational particle's process is the local or area impact velocity of gravity in the gravitational interactions field(s).

\subsubsection{Specification of Earth's Strength Limit by Earth's Gravity Variation with Altitude}

Cohesion constant limit number of the earth is derived based on effectiveness of earth's gravity variation with altitude as follows:

$$
X_{C \text {-earth }}=\frac{g_{h}}{\left(\frac{r_{e}}{r_{e}+h}\right)^{2} \times e^{e}}[41]=\frac{g_{h}}{\left(\frac{r_{e}}{r_{e}+h}\right)^{2}} \times \text { A.D }
$$

where $\boldsymbol{g}_{\boldsymbol{h}}$ is gravity at height $\boldsymbol{h}$ above sea level, $\boldsymbol{g}_{\mathbf{0}}$ is standard gravity, $\boldsymbol{r}_{\boldsymbol{e}}$ is radius of earth, $\boldsymbol{h}$ is height from sea level and A.D is area density [41].

\subsubsection{Role of Old and Accurate Age of the Earth in Strength Limit Calculation}

The strength limit of earth is used to predict exact age and decompose time of earth, stars, planets and galaxies. Exact age of the earth $\left(\mathbf{A O E}_{\text {Exact }}\right)$ shows that earth gravity $\left(10.2 \mathrm{~m} / \mathrm{s}^{2}\right)$ in 4.7303652362904 billion years ago when creation procedures of the earth started from the beginning time with approximately $0.673 \mathrm{MPa}$ strength limit value is much more than gravity value $\left(9.80665 \mathrm{~m} / \mathrm{s}^{2}\right)$ in 4.5501750498945017745329167415032 billion years ago (old age of the earth) after full creation of the earth with approximately $0.65 \mathrm{MPa}$ value of strength limit. In fact gravity value along the 180190186.4 years creation process of the earth from 4.7304 billion years ago to 4.56 billion years ago was higher than now and equal to $10.2 \mathrm{~m} / \mathrm{s}^{2}$.

Old and present strength limit of earth calculated directly from old and present age of the earth by follow relation: 


$$
\begin{aligned}
& \left.X_{C \text {-Earth,Old }}=\ln \left[\left(\frac{(\text { Age of the earth }}{\text { Old }}\right) \times \operatorname{VDR}_{X_{C \text {-Earth }}^{\text {Old }}}^{\text {PGCOTR }}\right)+1\right] \\
& =\ln \left[\left(\frac{\left(\mathrm{AOE}_{\text {Old }}\right) \times 2}{10^{10}}\right)+1\right] \\
& \left.X_{C \text {-Earth,Now }}=\ln \left[\left(\frac{(\text { Age of the earth }}{\text { Exact }}\right) \times \operatorname{VVR}_{X_{C \text {-Earth }}^{\text {Now }}}\right)+1\right] \\
& X_{C-\text { Earth,Now }}=\ln \left[\left(\frac{\left(\mathrm{AOE}_{\text {Exact }}\right) \times\left[\frac{1}{\text { A.D }}\right]^{0.26022305719176147124351862627535}}{\text { PGCOTR }}\right)+1\right]
\end{aligned}
$$

where [41] Age of the earth ${ }_{\text {Old }}$ or $\mathbf{A O E}_{\text {Old }}$ is age of the earth according to old standard gravity and equal to 4.5501750498945017745329167415032 billion years, $\boldsymbol{X}_{C \text {-Earth,old }}$ is the cohesion constant limit number of earth or strength limit parameter of earth or strength of existence global gravitation according to old standard gravity and equal to $0.64712157172243419172410449319661 \mathrm{MPa}$, PGCOTR or PGSCOTR is principle great changes occurrence time range in earth or principle great structural changes occurrence time range or time range of great structural variations occurrence possibility or further is changeable or mutation revolutionary span and equal to $10^{10} \mathrm{Year}, \mathbf{V D V R}_{X_{C-\text { Earth }}}^{\text {old }}$ is variations distance value range of earth's strength limit in 10 billion year according to old standard gravity and equal to $2 \mathrm{~km}$, Age of the earth Exact $_{\text {or }} \mathbf{A O E}_{\text {Exact }}$ is accurate or exact age of the earth according to present standard gravity at present time and equal to 4.7303652362904 billion years, $\boldsymbol{X}_{C-\text { Earth,Now }}$ is the cohesion constant limit number of earth or strength limit parameter of earth or strength of existence global gravitation according to present standard gravity and equal to $0.6727480488031732255208148942682 \mathrm{MPa}, \mathbf{V V R}_{X_{C-\text { Earth }}}^{\text {Now }}$ is variations value range of earth's strength limit in 10 billion year according to present standard gravity and equal to $2.0286278077335909781784347367861 \mathrm{~km}$ and A.D is area density or surface density (S.D or $\boldsymbol{D}_{\boldsymbol{S}}$ ) and equal to $0.06598803584531253707679018759685 \mathrm{Ggr} / \mathrm{m}^{2} \approx 0.066 \mathrm{Ggr} / \mathrm{m}^{2}$.

\subsubsection{Why Age of the Earth since Initial Time of Creation Procedures ( The Present Age of Earth $=4.7303652362904$ Billion Years) Is Exact for the Earth?}

Simple interpretation of this number $\left(\mathbf{A O E}_{\text {Exact }}=4.7303652362904\right.$ Billion Year) shows importance subjects. number 7 means: 7 skies, 7 spaces, 7 days in 1 week, 7 protecting layers of the earth's sky; number 30 means: 1 month have 30 days; number 365 means: 1 year is approximately equal to 365 days (exactly equal to 365.2362904 days); number 23 means: 23.62904 hours exist in 1 day; number 6 means: earth created in 6 cycles, number 2 means: 2 cycles exist in one day, day and night, 2 procedures exist for occurrence of 24 hours in one day; number 90 means: 1 season has 90 days; number 4 means: 4 seasons exist in a 
year and besides 4 cycles passover in earth's age.

Lifetime of one part of human's body dependent to overall age and lifetime of human also lifetime of earth dependent to lifetime of existence and universe.

\subsubsection{Strength Limit Definition by Précising a Previous Explanation Through Newton's Second Law of Motion and Newton's Law of Universal Gravitation and Standard Gravity}

Different calculated values offered for G, E, R and other specifications of earth. Strength limit find the accurate values for these parameters. Accordingly, there are more words to say via classical gravitations.

According to Newton's second law of motion [43] [44] and newton's law of universal gravitation between earth and every point mass on earth or in distance from earth [45] [46] [47] [48] [49] below relation is feasible:

$$
F=m a=m g=\frac{M \times G \times E}{R^{2}}
$$

Standard gravity then is given by below equation without deleting $m$ and $M$ from 2 sides of Equation (18):

$$
\begin{aligned}
& g_{0}=\frac{F}{m}=\frac{M}{m} \times \frac{G \times E}{R^{2}}=R^{\prime} \times \frac{G \times E}{R^{2}}=\frac{G \times E}{R^{\prime \prime}}=\frac{X_{C-\text { Earth }}}{\text { A.D }}=\frac{X_{C-\text { earth }}}{\frac{1}{e^{e}}} \\
& \frac{g_{0} \times R^{2}}{G}=E \times R^{\prime}=E D \times V_{\text {Earth }} \times R^{\prime} \\
& =242892793676594.55573513891084111 \mathrm{~kg} \cdot \mathrm{m}
\end{aligned}
$$

The ratio of every point mass $M$ to mass $m$ in calculations is equal to the distance between the masses only from quantitative aspect and can be written as:

$$
\begin{aligned}
& \frac{M}{m}=R^{\prime}=\frac{R_{\text {Earth }}^{2}}{R^{\prime \prime}}=\frac{g_{0} \times R_{\text {Earth }}^{2}}{G \times E}=40656957195372.527825506161635217 \\
& R^{\prime}=R_{\text {Earth-Dimensionless }}=\text { The Quantitative Value of } R=\text { Dimensionless } R \\
& \qquad R^{\prime \prime}=\frac{R_{\text {Earth }}^{2}}{R^{\prime}}=\frac{R_{\text {Earth }}^{2}}{\frac{M}{m}}
\end{aligned}
$$

Cohesion limit number of the earth with impact of earth density clearly expressed as:

$$
\begin{aligned}
X_{C-\text { Earth }} & =\frac{M}{m} \times \frac{G \times E}{R_{\text {Earth }}^{2}} \times \mathrm{A} . \mathrm{D}=\frac{G \times E}{R^{\prime \prime} \times e^{e}}=\frac{G \times E D \times V_{\text {Earth }}}{R^{\prime \prime}} \times \mathrm{A} . \mathrm{D} \\
& =0.64712157172243419172410449319661 \\
X_{C \text {-Earth }} & =G \times E D \times V_{\text {Earth }} \times \frac{R_{\text {Earth-Dimensionless }}}{R_{\text {Earth }}^{2}} \times \text { A.D } \\
& =G \times E D \times V_{\text {Earth }} \times \frac{R_{\text {Earth-Dimensionless }}}{R_{\text {Earth }}(\text { in } \mathrm{m}) \times R_{\text {Earth }}(\text { in } \mathrm{m})} \times \mathrm{A} . \mathrm{D} \\
& =\frac{G \times E D \times V_{\text {Earth }}}{R_{\text {Earth }}^{\prime}} \times \text { A.D }
\end{aligned}
$$


where $G$ is the gravitational constant or Newtonian constant of gravitation that's approximately equal to $6.67384 \times 10^{-11} \pm 0.00080 \times 10^{-11} \mathrm{~m}^{3} \mathrm{~kg}^{-1} \mathrm{~s}^{-2}$ [50] or $6.67384 \times 10^{-11} \mathrm{~N} \mathrm{~m}^{2} \mathrm{~kg}^{-2}$ [48] [51] [52], $E$ (also identified by $\mathcal{M}_{\oplus}$ ) is the mass of the earth that's equal to $\mathcal{M}_{\oplus}=5.9742 \times 10^{24} \mathrm{~kg}$ [53], $M$ is the every point mass (in $\mathrm{kg}$ ), $\boldsymbol{R}_{\text {Earth }}$ or $\boldsymbol{R}$ is the distance between the masses (in $\mathrm{m}$ ) which equal to $40,656,957,195,372.527825506161635217 \mathrm{~m}, E D$ is the earth density (in $\mathrm{kg} / \mathrm{m}^{3}$ ) that's equal to $5.5152740465837649209294596615615 \mathrm{gr} / \mathrm{cm}^{3}, \quad V_{\text {earth }}$ is the volume of earth (in $\mathrm{m}^{3}$ ) and equal to $1.08321 \times 10^{12} \mathrm{~km}^{3}$ or $1.08321 \times 10^{27} \mathrm{~cm}^{3}$ [53] $\left(2.59876 \times 10^{11} \mathrm{cu} \mathrm{mi}[54]\right), \boldsymbol{R}^{\prime}$ is the ratio of every point mass $M$ to mass $m$ and equal to $40,656,957,195,372.527825506161635217$ (dimensionless), $\boldsymbol{R}^{\prime \prime}$ is equal to $40,656,957,195,372.527825506161635217 \mathrm{~m}^{2}, \boldsymbol{R}_{\text {Earth-Dimensionless }}$ is the quantitative radius value of earth without unit (dimensionless) and $\boldsymbol{R}_{\text {Earth }}^{\prime}$ is equal to $40,656,957,195,372.527825506161635217 \mathrm{~m}^{2}$.

\subsection{Strength Limit of the Earth Computation by Distance That Light Travel in One Year}

Old and present strength limit of earth in accordance to direct proportionality of old and present age of the earth and distance that light travel in one year gets by:

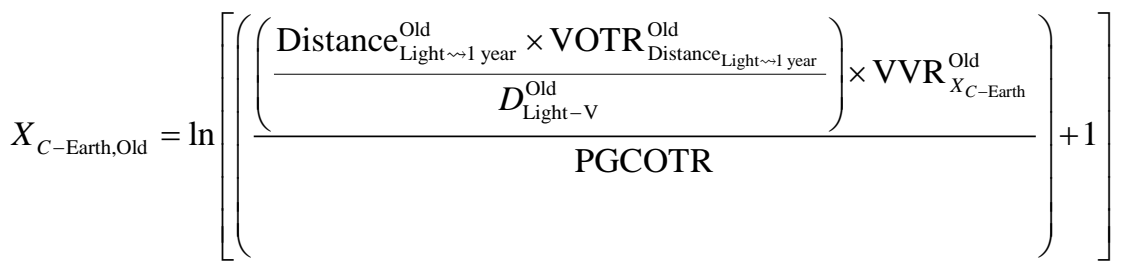

$X_{C-\text { Earth,Now }}=\ln \left[\left(\frac{\left(\frac{\text { Distance }_{\text {Light } w 1 \text { year }}^{\text {Now }} \times \mathrm{VOTR}_{\text {Distance }}^{\text {Lightw } w \text { year }}}{D_{\text {Light-V }}^{\text {Now }}}\right) \times \operatorname{VVR}_{X_{C-\text { Earth }}}^{\text {Now }}}{\text { PGCOTR }}\right)+1\right]$

where [41] Distance ${ }_{\text {Light } \sim 1 \text { year }}^{\text {Old }}$ is value of distance that light travel during one year according to old standard gravity and equal to 9.1003500997890035490658334830064 $\times 10^{12} \mathrm{~km}, \quad \boldsymbol{D}_{\text {Light-v }}^{\text {Now }}$ is Variations rate of distance that light travel in 0.001 year or 0.36525 day or 8.784 hour or 527.04 Min or 31622.4 second according to old standard gravity and equal to $2 \mathrm{Km}$, VOTR $_{\text {Distance }}^{\text {Light } \sim 1 \text { year }}$ is Variations occurrence time range of distance that light travel in one year according to old standard gravity and equal to $10^{-3}$ year. This means time range of occurrence of distance variations is $10^{-3}$ year in light travel during one year according to old standard gravity (distance variations during light travel in one year occur in $10^{-3}$ year), Distance $\mathrm{LLight} \sim 1$ year $_{\text {Now }}$ is value of distance that light travel in one year according to present standard gravity and equal to $9.460730472580800 \times 10^{12} \mathrm{~km}$, $D_{\text {Light-V }}^{\text {Now }}$ is Variations rate of distance that light travel in one year according to present standard gravity in $1.0143139038667954890892173683931 \times 10^{-3}$ year

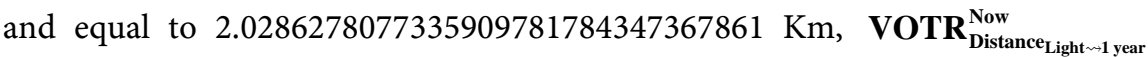


is Variations occurrence time range of distance that light travel in one year according to present standard gravity based on 10 billion year principle great structural changes occurrence time range in a defined variations rate of $2.0286278077335909781784347367861 \mathrm{Km}$ and equal to $1.0143139038667954890892173683931 \times 10^{-3}$ year and PGCOTR or PGSCOTR is Principle great structural changes occurrence time range in earth or time range of great structural variations occurrence possibility or in addition is changeable or mutation revolutionary span and equal to $10^{10}$ Year.

\subsection{Earth's Strength Limit Computing by Thickness of Homosphere}

Thickness of homosphere and its varieties leads to calculate the old and present strength limit of earth from following relations:

$$
\begin{aligned}
& X_{\text {C-Earth,Old }}=\ln \left[\left(\frac{\left(\frac{\mathrm{TH}_{\text {Old }} \times \mathrm{VTR}_{\text {th }}^{\text {Old }}}{\mathrm{TH}_{\mathrm{V}}^{\text {Old }}}\right) \times \mathrm{VVR}_{X_{C-\text { Earth }}^{\text {Old }}}^{\text {PGCOTR }}}{\text { PGCOT }}\right]+1\right]
\end{aligned}
$$

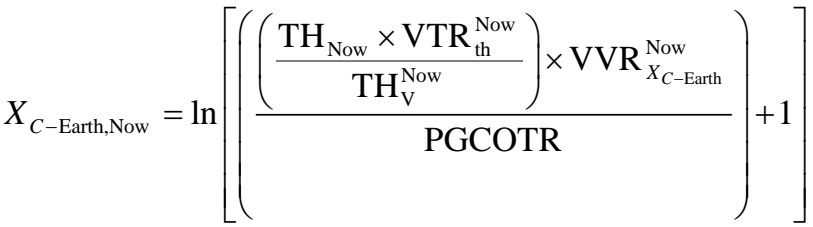

where [41] $\mathbf{T H}_{\text {old }}$ is value of thickness of homosphere according to old standard gravity in variation time range of 100 million year and equal to $91.003500997890035490658334830064 \mathrm{Km}, \mathbf{T H}_{\text {Now }}$ is value of thickness of homosphere according to present standard gravity in variation time range of $101,431,390.38667954890892173683931$ million year and equal to

$94.60730472580800 \mathrm{Km}$ and exactly equal to 94.6073047258079999999999999999995 $\mathrm{Km}, \mathbf{T H}_{\mathrm{v}}^{\text {Old }}$ is Thickness of homosphere variations rate in occurrence range (possibility occurrence range) of 100 million year according to old standard gravity and equal to $2 \mathrm{Km}, \mathbf{T H}_{\mathbf{v}}^{\text {Now }}$ is Thickness of homosphere variations rate in occurrence range (possibility occurrence range) of 101,431,390.38667954890892173683931 million year (in a defined variations time range) according to present standard gravity and equal to $2.0286278077335909781784347367861 \mathrm{Km}, \mathbf{V T R}_{\text {th }}^{\text {Old }}$ is Variations time range of thickness of homosphere according to old standard gravity and equal to $10^{8}$ year and $\mathbf{V T R}_{\text {th }}^{\text {Now }}$ is Variations time range of thickness of homosphere according to present standard gravity in a defined variations rate and equal to $1.0143139038667954890892173683931 \times 10^{8}$ year.

\subsection{Determination of Strength Limit with Effective Radius of Earth; One New Importance Parameter}

Effective radius of earth in gravitational field or in forces equivalent fields (equivalent fields between forces) is the effective regional radius that's cosmos forces impact on it with steady state equilibrium. In fact this factor is an incons- 
picuous part of sided air streamer. This radius is a part of inconspicuous pillar between spaces or galactic inconspicuous pillar or inters spaces inconspicuous pillar or space medial pillar. Earth's strength limit number that shows the structural strength of gravitational field in stable state of equilibrium is determined by the following formulas:

$$
\begin{aligned}
X_{C \text {-Earth }} & =\frac{E R_{\text {earth }}}{\mathrm{DLSLC}}=\frac{\text { Effective Radius of Earth }}{10^{4}} \\
& =E R_{\text {earth }} \times \mathrm{SLCER}=E R_{\text {earth }} \times 10^{-4} \\
X_{C \text {-Earth,Old }} & =\frac{E R_{\text {earth,Old }}}{\mathrm{DLSLC}}=\frac{\text { Effective Radius of Earth }}{10^{4}} \\
& =E R_{\text {earth,Old }} \times \mathrm{SLCER}=E R_{\text {earth,Old }} \times 10^{-4} \\
X_{C \text {-Earth,Now }} & =\frac{E R_{\text {earth,Now }}}{\text { DLSLC }}=\frac{\text { Effective Radius of Earth }}{10^{4}} \\
& =E R_{\text {earth,Now }} \times \operatorname{SLCER}=E R_{\text {earth,Now }} \times 10^{-4}
\end{aligned}
$$

where SLCER is the strength limits changes in accordance to every kilometer of earth's radius and equal to $10^{-4} \mathrm{MPa} / \mathrm{km}, \boldsymbol{E} \boldsymbol{R}_{\text {earth }}$ is the effective radius of earth and equal to $6471.2157172243419172410449319661 \mathrm{~km}$ and DLSLC is the distance limits of strength limit changes or distance limits in accordance to every 1 $\mathrm{MPa}$ of strength limits changes and equal to $10^{4} \mathrm{~km} / \mathrm{MPa}$.

Effective radius of earth or earth's effective radius according to old and present strength limit of earth are calculated by follow relations:

$E R_{\text {earth,Old }}=X_{C-\text { Earth,Old }} \times$ SLCER $=6471.2157172243419172410449319661 \mathrm{~km}$

$E R_{\text {earth,Now }}=X_{C-\text { Earth,Now }} \times \mathrm{SLCER}=6727.480488031732255208148942682 \mathrm{~km}$

where $\boldsymbol{E R}_{\text {earth,old }}$ is the effective radius of earth according to old strength limit of earth and equal to $6471.2157172243419172410449319661 \mathrm{~km}$ and $\boldsymbol{E}_{\text {earth,Now }}$ is the effective radius of earth according to present strength limit of earth and equal to $6727.480488031732255208148942682 \mathrm{~km}$.

\subsection{Impacts of Molten Particle (or U.C.F.P or C.C.F.P) Characteristics in Strength Limit Specifications}

Strength limit of earth help to discover fundamental and basis particles (particles in ultra-time dimensions also parallel particles and parallel characteristics of particles). Particles create energies and energies organize behaviors. U.C.F.P or C.C.F.P particles are unidentified continued flowing (fluxing) particles or complex combined fluxing particles (complex combined continued flowing particles). This particle is an infinitesimal basis structural particles or infinitude fundamental particles or molten particles with hyper-extreme energy. Strength limit of earth have relevance to structural particles and their structures. These energetic particles forms earth and universe so have ultra-high seismo galactic energy and identify in $10^{(-124)}$ scaling. So, they have importance role in genesis, 
forming and decomposing of earth and universe and knowing them has significant effects on basis works. This particle will use to find genesis of universe, galaxies, stars and other parts of existence and to make new extra intelligent computer and hyper speedy and accurate telecommunication systems. Additionally use to create neutral cover for restrain from nuclear destroyer actions. This antinuclear rocket defense shield is impenetrable. Atomic weight and speed of molten particle are as follows:

Atomic weight of U.C.F.P or C.C.F.P.

$$
\begin{aligned}
& =A W_{\text {C.C.F.P }}=\left[4+\left(\left(\frac{V_{\text {C.C.F.P-Now }}}{\text { C.C.F.P. }}\right)-3\right)\right]=\frac{e}{X_{\text {C-Earth }}} \\
& A W_{\text {C.C.F.P-Old }}=\left[4+\left(\left(\frac{V_{\text {C.C.F.P-Old }}}{\text { C.C.F.P }}\right)-3\right)\right]=\left[4+\left(\left(V_{\text {C.C.F.P-Old }} \times 10^{-8}\right)-3\right)\right] \\
& =\frac{e}{X_{C-\text { Earth,Old }}}=\frac{\ln \left(\frac{1}{\text { A.D }}\right)}{X_{C-\text { Earth,Old }}} \\
& A W_{\text {C.C.F.P-Now }}=\left[4+\left(\left(\frac{V_{\text {C.C.F.P-Now }}}{\text { C.C.F.P.P }}\right)-3\right)\right] \\
& =\left[4+\left(\left(V_{\text {C.C.F.P-Now }} \times \frac{1}{10^{8}}\right)-3\right)\right]=\frac{\ln \left(\frac{1}{\text { A.D }}\right)}{X_{\text {C-Earth,Now }}}
\end{aligned}
$$

where $A W_{\text {C.C.F.P-Old }}$ is the Atomic weight of C.C.F.P according to old strength limit of earth and equal to $4.2005736591716971201366713081801, V_{\text {C.C.F.P-Old }}$ is the C.C.F.P particles speed according to old strength limit of earth and equal to $3.2005736591716971201366713081801 \times 10^{8} \mathrm{~m} / \mathrm{s}, \quad \boldsymbol{A} \boldsymbol{W}_{\text {C.C.F.P-Now }}$ is the atomic weight of C.C.F.P according to present strength limit of earth and equal to $4.0405644182765017530136549521107, \quad V_{\text {C.C.F.P-Now }}$ is the C.C.F.P particles speed according to present strength limit of earth and equal to

$3.0405644182765017530136549521107 \times 10^{8} \mathrm{~m} / \mathrm{s}$ and C.C.F.P $P_{\text {UV }}$ is the unit velocity in measurement of complex combined fluxing particles' features based on old and present strength limit of earth and equal to $10^{8} \mathrm{~m} / \mathrm{s}$.

Speed of C.C.F.P particle is faster than speed of light phenomenally because provide unite energies of composing components, particles and elements of universe. C.C.F.P particle's characteristics changing during time lapses according to old and present value of strength limit of earth and standard gravity. Gravity is a little sign of equivalency. Effective gravity between mother particles to create generation energy is one of the effective absorption impacts between them. Absorption gravity of same mother particles to creates generation energies determined by specifications of them with having pattern in time at regular and irregular intervals.

Then cohesion constant limit number of the earth in general, old and present 
forms are written by below formulas:

$$
\begin{gathered}
X_{\text {C-Earth }}=\frac{e}{4+\left(\frac{\text { molten particle speed }}{\text { Unit Velocity }}-3\right)}=\frac{\ln \left(\frac{1}{A . D}\right)}{\text { Atomic weight of C.C.F.P }} \\
X_{\text {C-Earth,Old }}=\frac{e}{A W_{\text {C.C.F.P-Old }}}=\frac{\ln \left(\frac{1}{A . D}\right)}{\left[4+\left(\left(\frac{V_{\text {C.C.F.P-Old }}}{\text { C.C.F.P. }}\right)-3\right)\right]} \\
X_{\text {C-Earth,Now }}=\frac{e}{A W_{\text {C.C.F.P-Now }}}=\frac{\ln \left(\frac{1}{A . D}\right)}{\left[4+\left(\left(\frac{V_{\text {C.C.F.P-Now }}}{\text { C.C.F.P. }}\right)-3\right)\right]}
\end{gathered}
$$

Thereupon, we get Euler's number according to Earth's strength limit and C.C.F.P particles as:

$$
\begin{aligned}
& e=X_{C \text {-Earth }} \times\left[4+\left(\left(\text { U.C.F.P speed } \times 10^{-8}\right)-3\right)\right] \\
& e=X_{\text {C-Earth,Old }} \times\left[4+\left(\left(\frac{\text { U.C.F.P speed }}{\text { old }}\right)-3\right)\right] \\
& e=X_{C-\text { Earth,Now }} \times\left[4+\left(\left(\frac{{\text { U.C.F.P } \text { speed }_{\text {now }}}_{\text {C.C.F.P. }}}{\mathrm{UV}}\right)-3\right)\right]
\end{aligned}
$$

where U.C.F.P speed old $_{\text {or }}$ or $\boldsymbol{V}_{\text {C.C.F.P-old }}$ is the C.C.F.P particles speed according to old strength limit of earth and U.C.F.P speed ${ }_{\text {now }}$ or $\boldsymbol{V}_{\text {C.C.F.P-Now }}$ is the C.C.F.P particles speed according to present strength limit of earth.

According to above equations must ask this question: Is Napier's number constant or changing with variation of strength limit and C.C.F.P particle's specifications and make another new fundamental number too?

\subsection{Use of Age of the Galaxy in Strength Limit Definition}

The Universe and Milky Way Galaxy have greater ages than the Earth and Solar System's ages via consistent evidences from their antiquity [55].

a) The age of the Universe can be estimated in range from 7 to 20 billion years through the velocity and distance of galaxies as the universe expands. This estimate depending on whether the expansion is constant or is slowing due to gravitational attraction.

b) The age of the Galaxy is estimated to be $14-18$ billion years from the rate of evolution of stars in globular clusters, which are thought to be the oldest stars in the Galaxy. The age of the elements in the Galaxy, based on the production ratios of osmium isotopes in supernovae and the change in that ratio over time 
due to radioactive decay, is 8.6 - 15.7 billion years. Theoretical considerations indicate that the Galaxy formed within a billion years of the beginning of the Universe.

The best and the most consistent age for the universe are estimated to be 14 17 billion years by combining data.

Age of the Galaxy have direct proportional to age of the earth and number of day in one year. Age of the Galaxy is obtained by age of the earth which given as follows:

$$
\begin{gathered}
\mathrm{AOG}=\left[(\mathrm{AOE})_{\text {In billion year and Convert to second }}\right]=\left[\frac{\mathrm{AOE} \times 366 \times 60 \times 60 \times 24}{\text { Time }_{\mathrm{VL}}}\right] \\
\mathrm{AOG}_{\text {Old }}=\frac{\left[\left(\mathrm{AOE}_{\text {Old }}\right)_{\text {In billion year and Convert to second }}\right]}{\text { Time }_{\mathrm{VL}}}=\frac{\left[\mathrm{AOE}_{\text {Old }} \times 366 \times 24 \times 60 \times 60\right]}{10^{-2}} \\
\mathrm{AOG}_{\text {Old }}=14388745549.778389291498970636651 \text { years } \\
\mathrm{AOG}_{\text {Now }}=\frac{\left[\left(\mathrm{AOE}_{\text {Exact }}\right)_{\text {In billion year and Convert to second }}\right]}{\text { Time }_{\mathrm{VL}}} \\
=\left[\mathrm{AOE}_{\text {Exact }} \times 366 \times 24 \times 60 \times 60\right] \times 10^{2} \\
\mathrm{AOG}_{\text {Now }}=\mathrm{AOG}_{\text {Exact }}=14958550164.806954496 \text { years }
\end{gathered}
$$

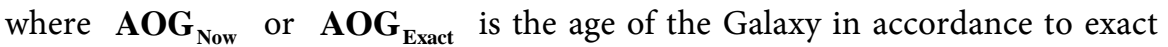
age of the earth and present strength limit of earth or in the other words is the age of the universe after 4.7303652362904 billion years from initial start time of earth creation in years. $\mathbf{A O G}_{\text {old }}$ is the age of the Galaxy based on old age and strength limit of the earth or is the age of the Galaxy after 4.5501750498945017745329167415032 billion years from full creation time of the earth in years and Time $_{\mathrm{VL}}$ is the time variation limit of age of the earth in accordance to every 1 year of age of the galaxy and equal to $10^{-2} \mathrm{~S} /$ Year.

The difference between these calculated ages of the Galaxies identify the exact time of big structural changes for earth in the past (identifies peak of changes points). In fact the difference between age of the Galaxy in accordance to present strength limit (also exact age) of the earth and age of the Galaxy based on old strength limit of earth characterize the last great climate changes specially ice ages for the earth and continental changes too. The last great super climate changes on earth occur in 569,806,615.028565204501029363349 years ago.

$$
\begin{aligned}
& \text { Difference of age of the galaxies }=\mathrm{AOG}_{\text {Exact }}-\mathrm{AOG}_{\text {Old }} \\
& =0.569806615028565204501029363349 \text { billion years }
\end{aligned}
$$

Thus, age of the earth in general, old and present forms is calculated based on old and present strength limit of earth and gravity according to above formulas becomes: 


$$
\begin{gathered}
\mathrm{AOE}=\frac{\mathrm{AOG} \times \mathrm{Time}_{\mathrm{VL}}}{366 \times 60 \times 60 \times 24}=\frac{\mathrm{AOG} \times 10^{-2}}{366 \times 24 \times 60 \times 60} \\
\mathrm{AOE}_{\text {Old }}=\frac{\mathrm{AOG}_{\mathrm{Old}} \times \mathrm{Time}_{\mathrm{VL}}}{366 \times 24 \times 60 \times 60}=\frac{\mathrm{AOG}_{\text {Old }}}{10^{2} \times 366 \times 24 \times 60 \times 60} \\
\mathrm{AOE}_{\text {Exact }}=\frac{\mathrm{AOG}_{\text {Now }} \times \mathrm{Time}_{\mathrm{VL}}}{366 \times 24 \times 60 \times 60}=\frac{\mathrm{AOG}_{\text {Now }}}{10^{2} \times 366 \times 24 \times 60 \times 60}
\end{gathered}
$$

Otherwise, old and present strength limit of the earth are defined based on old and present age of the earth and the Galaxy as:

$$
\begin{aligned}
& \left.X_{\text {C-Earth,Old }}=\ln \left[\left(\frac{(\text { Age of the earth }}{\text { Old }}\right) \times \operatorname{VVR}_{X_{C-\text { Earth }}}^{\text {Old }}\right)+1\right] \\
& =\ln \left[\left(\frac{\left(\frac{\text { AOG }_{\text {Old }} \times \text { Time }_{\mathrm{VL}}}{366 \times 24 \times 60 \times 60}\right) \times \mathrm{VVR}_{X_{C \text {-Earth }}}^{\text {Old }}}{\text { PGCOTR }}\right)+1\right] \\
& \left.X_{C-\text { Earth,Now }}=\ln \left[\left(\frac{(\text { Age of the earth }}{\text { Exact }}\right) \times \operatorname{VVR}_{X_{C-\text { Earth }}^{\text {Now }}}^{\text {PGCOTR }}\right)+1\right] \\
& =\ln \left[\left(\frac{\left(\frac{\mathrm{AOG}_{\text {Now }} \times \mathrm{Time}_{\mathrm{VL}}}{366 \times 24 \times 60 \times 60}\right) \times \mathrm{VVR}_{X_{C-\text { Earth }}}^{\text {Now }}}{\text { PGCOTR }}\right)+1\right]
\end{aligned}
$$

where $\mathbf{A O G}_{\text {Old }}$ is the age of the Galaxy since old age of the earth in year,

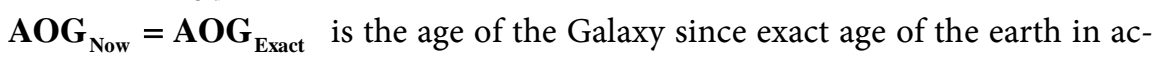
cordance to present strength limit of the earth in year and $\mathbf{A O G}_{\text {Exact }}$ is Accurate or exact age of the earth according to present standard gravity at present time in billion years.

$3.16224 \mathrm{sec}$ in $1 \mathrm{~s}=$ converter factor which convert age of the earth directly to age of the Galaxy

Another simplest form to shows the connections between old and present age of the earth to old and present age of the Galaxy is:

$$
\begin{aligned}
\mathrm{AOG}_{\mathrm{Old}} & =\left[\mathrm{AOE}_{\mathrm{Old}} \times \text { Conversion Factor }\right] \\
\mathrm{AOG}_{\text {Now }} & =\left[\mathrm{AOE}_{\text {Exact }} \times \text { Conversion Factor }\right]
\end{aligned}
$$

where $\mathbf{A O G}_{\text {Old }}$ is the age of the Galaxy based on old age and strength limit of the earth in billion years and $\mathbf{A} \mathbf{O G}_{\text {Now }}$ or $\mathbf{A O E} \mathbf{E}_{\text {Exact }}$ is the age of the Galaxy in accordance to present strength limit and age of the earth in billion years.

Conversion factor in above equation is a changing factor which connects age of the earth to age of the Galaxy and calculates as: 
Conversion Factor

$$
\begin{aligned}
& =10^{-7} \text { Year } / \mathrm{S}=0.0000001 \times 366 \times 24 \times 60 \times 60 \text { Second } / \text { Second } \\
& =3.16224 \text { dimensionless }
\end{aligned}
$$

Conversion factor furthermore specifies through unit of time as:

$$
\begin{aligned}
\text { Conversion Factor } & =\text { Unit Of Time } \div 10^{\mathrm{ECC}} \\
& =\left(\frac{1}{10} \times \text { number of seconds in one year }\right) \div 10^{6}
\end{aligned}
$$

In fact unit of time is equal to 10 percent of number of seconds in one year.

UOT $=$ Unit Of Time $=3162240$ seconds $=\frac{1}{10} \times$ number of seconds in one year

UOT $=10 \%$ of number of seconds in one year $=\frac{1}{10}$ number of seconds in 366 days

Unit of time distinguished by variations ranges as:

$$
\mathrm{UOT}=\left(\left[\operatorname{VOTR}_{\text {Distance }}^{\text {Olightw 1 year }}\right]_{\text {convert to hour }}\right)_{\text {in hour }} \times\left(10^{\mathrm{VDVR}_{X_{C} \text {-Earth }}^{\text {Old }}}\right)_{\text {in Sec/hour }} \times 60^{2}
$$

Unit of time calculated from multiplication of sequence of converting units in life time of gravitational particles which gets by:

$$
\mathrm{UOT}=(\mathrm{LTGP})_{\text {in seconds }} \times 60^{2}
$$

where [41] Nosioney is number of seconds in one year and equal to 31622400 second, VOTR $_{\text {Distance }_{\text {Light } w 1 \text { year }}}^{\text {Old }}$ is Variations occurrence time range of distance that light travel in one year according to old standard gravity and equal to 8.784 hour, $\mathbf{V D V R}_{X_{\text {C-Earth }}}^{\text {Old }}$ is Variations distance value range of earth's strength limit in 10 billion year according to old standard gravity and equal to $2 \mathrm{Km}$, LTGP is life time of gravitational particles and equal to 878.41046115788314777747042900908 Sec and ECC is the number of earth's creation cycles (number of cycles in earth creation) and equal to 6 cycles.

ECC is measured directly by below relations [41]:

$$
\begin{aligned}
& 10^{\mathrm{ECC}}=\left[\frac{1}{\left(\operatorname{VOTR}_{\text {Distance } \left._{\text {Lightw1 year }}\right)_{\text {in year }}}^{\text {Old }}\right.}\right]^{\mathrm{VDVR}_{X_{C-\text { Earth }}}^{\text {Old }}}
\end{aligned}
$$

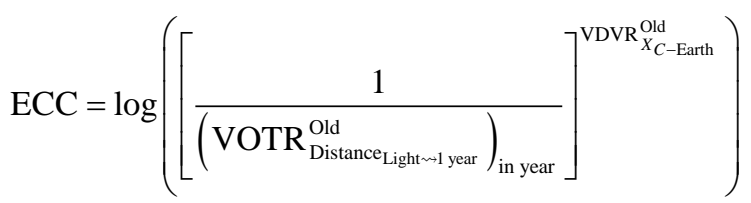

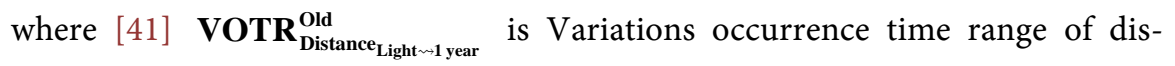


tance that light travel in one year according to old standard gravity and equal to $10^{-3}$ year (distance variations during light travel in one year occur in $10^{-3}$ year) and $\mathbf{V D V R}_{X_{C-\text { Earth }}}^{\text {Old }}$ is Variations distance value range of earth's strength limit in 10 billion year according to old standard gravity and equal to $2 \mathrm{Km}$.

\subsection{Feature of $X_{C \text {-Earth }}$ by Number of Cells in a Body}

A current estimation of human total cell number calculated for a variety of organs and cell types correspond to a total number of $3.72 \times 10^{13}$. This presented cell count could be a starting point for a common effort to complete the total calculation [56]. Age of the earth is considerable in various points of approaches like human body in levels of their life; before creation process, beginning time of creation process, creation procedures, whole creation procedures, whole creation point, since beginning of whole creation until now. Age of the earth is obtained by below formula in accordance to the total number of cells in human body as:

$$
\begin{gathered}
\mathrm{AOE}=\left[\left(\mathrm{NCB} \times 10^{-13}\right)-3\right]+4 \\
\mathrm{AOE}_{\text {Exact }}=\left[\left(\mathrm{NCB} \times 10^{-13}\right)-3\right]+4
\end{gathered}
$$

where NCB is the total number of cells in human body.

Human total cell number has direct proportion to age of the earth and increased with increment of age of the earth. The exact value of NCB is $3.7303652362904 \times 10^{13}$. This important issue demonstrates that lapse of time happened to put human kind on earth for live after initial and full creation of earth. Because adequate live conditions should form for exist human being on earth. Number of cells in human's body should have unison with age of the earth. This means that human appear on earth when NCB concordance with conditions and age of the earth. Thus can respond to some fundamental key questions in accordance to these features like as: When was the first presence of humankind on earth?

Furthermore shape of the universe can be imaginable by understand the procedures of creation to perfection of earth's and human being living. The universe expansion rate has direct proportion with total number of cells in human body in accordance to the following relation:

$$
\mathrm{NCB}=\left(\frac{\text { Universe's Expansion Rate }}{\mathrm{ER}_{\mathrm{UNIT}}}+3\right) \times 10^{13}
$$

where the universe expansion rate is equal to $73.03652362904 \mathrm{~km} / \mathrm{s}$ Megaparsec, $\mathbf{E R}_{\text {UNIT }}$ is the unit expansion rate range in 1 billion year and equal to 100 $\mathrm{km} /$ Second Megaparsec billion year, $10^{13}$ value is in $1 /$ billion year.

\subsection{Definitions of $X_{C \text {-Earth }}$ from Different Approaches}

Earth's strength limit factor has more scientific definitions from different approaches based on importance determinant features that shown in Table 1. 
Table 1. Different definitions of strength limit number of earth according to old and present standard gravity.

Strength limit number of earth calculation formulas based on importance determinant features Equations NO. Determination of $\boldsymbol{X}_{\mathrm{C}-\mathrm{E} \text { arth }}$ with thickness of homosphere

$$
\begin{gathered}
X_{C \text {-Eart }}=\ln \left(\frac{\text { Thickness of homosphere }}{100}+1\right)=\ln \left(\frac{T H_{\text {homosphere }}}{100}+1\right) \\
X_{C-\text { Earth }}=\frac{\log \left(\frac{\text { Thickness of homosphere }}{100}+1\right)}{\log (e)}=\frac{\log \left(\frac{\text { Thickness of homosphere }}{100}+1\right)}{0.43429448190325182765112891891661}
\end{gathered}
$$

Determination of $\boldsymbol{X}_{\mathrm{C-Earth}}$ with distance that light travel along one year

$$
\begin{aligned}
& X_{C \text {-Earh }}=\ln \left[\left(\frac{\text { Distance that light travel along one year }}{10^{13}}\right)+1\right]=\ln \left[\left(\frac{\text { Distance }_{\text {Ligh } \sim \text { year }}}{10^{13}}\right)+1\right] \\
& X_{C \text {-Earth }}=\frac{\log \left(\frac{\text { Distance }_{\text {Lighw } \sim \text { year }}}{10^{13}}+1\right)}{\log (e)}=\frac{\log \left(\frac{\text { Distance }_{\text {Lightw } w \text { year }}}{10^{13}}+1\right)}{0.43429448190325182765112891891661}
\end{aligned}
$$

Determination of $\boldsymbol{X}_{\text {C-Earth }}$ with 1 Parsec value and Distance Light $\sim 1$ year $\sim 1$ year $_{\text {. }}$

$$
\begin{aligned}
& X_{\text {C-arth oLD }}=\ln \left(\frac{\text { Distance }_{\text {Lighw }-1 \text { year }}^{\text {OLD }} \times 3.0739148433095432861689099115875}{1 \mathrm{PC}_{\text {in km-Old }}}+1\right) \\
& X_{C \text {-earth Eaac }}=\ln \left(\frac{\text { Distance }_{\text {Lightw1 year }}^{\text {Now }} \times 3.2}{1 \mathrm{PC}_{\text {in km-Now }}}+1\right)
\end{aligned}
$$

Determination of $X_{C-E a r t h}$ with 1 Parsec value in miles

$$
\begin{aligned}
& X_{C-\text {-earth oLD }}= \ln (1.9100350099789003549065833483006) \\
& X_{C \text {-eart }}=\ln (1.9596150459074984227984337103851) \\
& X_{C \text {-Earth }}=\ln \left(\frac{1 \text { Parsec in miles }}{10^{13}}\right)
\end{aligned}
$$

Determination of $X_{C-E a r t h}$ with a closest number to 1

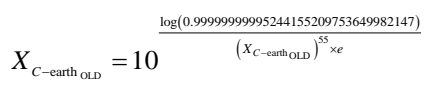

Determination of $X_{C-E a r h}$ with equatorial radius of earth

$$
X_{\text {C-Earth }}=\operatorname{Arccos}\left[\frac{1}{\left(R_{\text {EQUATOR }} \times 10^{-8}\right)+1}\right]
$$

Determination of $\boldsymbol{X}_{C-E a r h}$ with earth density

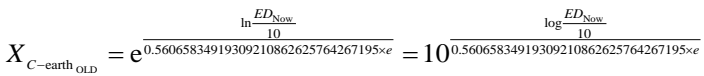

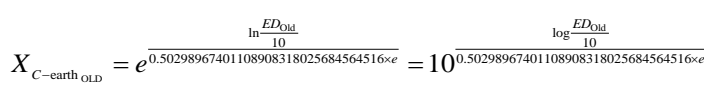

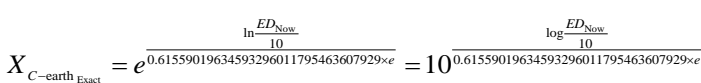

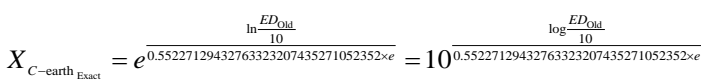

$$
\begin{aligned}
& X_{C \text {-earth oLD }}=\frac{g_{0 \text {-Old }}}{100\left(E D_{\text {Now }}-5\right)}
\end{aligned}
$$

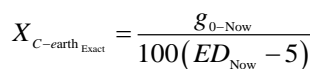

Determination of $X_{C \text {-Earth }}$ with xenon gas's percent of volume in dry atmosphere

$X_{C \text {-Earh }}=\left(\right.$ Volume percent of xenon gas in dry atmosphere $\times$ orbital inclination to sun's equator $\left.\times 10^{4}\right)$ 


\section{Results}

\subsection{Displaying Invisible Changes of Some Importance Features of Earth with Strength Limit of Earth}

Earth's strength limit, earth gravity, thickness of homosphere, distance that light travel during one year, volume percent of helium and xenon gas in air, effective and equatorial radius, equatorial diameter and orbital inclination to sun's equator and general radius, diameter, volume and density had higher amounts in the beginning time of the earth's creation process in 4.7303652362904 billion years ago (present, now or new calculated historical values) in comparison with 4.5501750498945017745329167415032 billion years ago after full earth's creation. In fact these specifications during the 180,190,186.4 years earth's creation process from 4.7304 billion years ago to 4.56 billion years ago demonstrate higher values than now. But earth's area density shows apparent constant behaviour in these areas. Thus must be defining features of earth according to these instant variations. Various importance features of earth and universe based on previous knowledge, adjusted old and present strength limit number and gravity of earth furthermore their related equations shows in Tables 2-5. The comparison between old and new peered values of earth and universe's features which represent extremely small impalpable variations of these specifications during life time of universe and earth are shown in Figure 1.

Table 2. Various calculated features of earth based on previous knowledge ${ }^{\mathrm{a}}$.

\begin{tabular}{|c|c|c|}
\hline Descriptions & Abbreviations & Adjusted values \\
\hline Earth Mean density & $\bar{\rho}_{\oplus}$ & $5.515 \mathrm{~g} / \mathrm{cm}^{3}[53]$ \\
\hline Distance value that light travel along one year & Distance $_{\text {Light } \rightsquigarrow 1 \text { year }}$ & $9.460730472 \times 10^{12} \mathrm{~km}[53]$ \\
\hline 1Parsec in miles & $1 \mathrm{Pc}_{\text {in miles }}$ & 19.174 Trillion miles $=1.9174 \times 10^{13}$ miles $[53]$ \\
\hline 1Parsec in light year & $1 \mathrm{PC}_{\text {in light year }}$ & 3.261633 Light year [53] \\
\hline Standard gravity of earth & $g_{0}$ & $980.665 \mathrm{~cm} / \mathrm{s}^{2} \cong 9.80665 \mathrm{~m} / \mathrm{s}^{2}[56]$ \\
\hline Thickness of homosphere & $\mathrm{TH}$ & $80-100 \mathrm{~km}[57][66]$ \\
\hline 1 Parsec in $\mathrm{km}$ & $1 \mathrm{Pc}_{\mathrm{inkm}}$ & $\begin{array}{l}30.8567758149137 \text { trillion kilometers }[58] \cong 3.0856776 \times 10^{16} \text { meters } \\
{[53]}\end{array}$ \\
\hline Sidereal rotation period & SRP & 0.99726968 d (23 h 56 m $4.100 \mathrm{~s})$ [53] [58] \\
\hline Equatorial diameter & $D_{\text {earth-Equatorial }}$ & $12,756.2732 \mathrm{~km} \cong 12,756.274[50][59]$ \\
\hline Equatorial radius & $R_{\text {EQUATOR }}$ & $\begin{array}{l}6,378,136.6 \pm 0.1 \mathrm{~m}[50][59]=6,378.1366 \mathrm{~km}=6.3781366 \times 10^{6} \mathrm{~m} \\
{[60] \cong 6,378,137 \mathrm{~m}[50][59]}\end{array}$ \\
\hline Speed of light & C & $299,792.458 \mathrm{~ms}^{-1}=2.99792458 \times 10^{8} \mathrm{~ms}^{-1}[48][50][51][52][60]$ \\
\hline Earth density & $E D$ & $5.514 \mathrm{gr} / \mathrm{cm}^{3}[54][61]$ \\
\hline Volume percent of helium gas in dry atmosphere & $V P_{\text {Helium gas-dry atm. }}$ & $\begin{array}{l}0.000524 \%=5.24 \mathrm{e}-4 \% \\
5.2 \text { parts per million }[62][63]\end{array}$ \\
\hline Volume percent of xenon gas in dry atmosphere & $V P_{\text {Xenon gas-dry atm. }}$ & $\begin{array}{l}0.0000087 \% \text { Percent by volume } \\
87 \pm 1 \text { parts per billion }(\mathrm{nL} / \mathrm{L})[64]\end{array}$ \\
\hline Diameter of earth & $D_{\text {earth }}$ & $12,742 \mathrm{~km}[65]$ \\
\hline Earth radius (Mean radius) & $R_{\oplus}$ & $6,371 \mathrm{~km}(3958.8 \mathrm{mi})[65]$ \\
\hline Earth's orbital inclination to sun's equator & $O I_{\text {to sun's equator }}$ & $7.155^{\circ}[66]$ \\
\hline Age of the primordial earth & $\mathrm{AOE}$ & 4.54 billion years $4.54 \pm 0.04$ Gya [67] [68] [69] [70] \\
\hline
\end{tabular}

aThese values established based on past knowledge from earth. 
Table 3. Adjusting various features based on old strength limit number of earth ${ }^{\mathrm{b}}$.

\begin{tabular}{|c|c|c|}
\hline Descriptions & Abbreviations & Adjusted values \\
\hline Old Thickness of homosphere & $T H_{\text {OLD }}$ & $91.003500997890035490658334830064 \mathrm{Km}$ \\
\hline Old Distance value that light travel along one year & Distance $_{\text {Lightw1 year }}$ & $9.1003500997890035490658334830064 \times 10^{12} \mathrm{Km}$ \\
\hline Old 1Parsec in Km & $1 \mathrm{Pc}_{\text {in km-old }}$ & 30.739148433095432861689099115876 trillion kilometers \\
\hline Old 1 Parsec in miles & $1 \mathrm{Pc}_{\text {in miles-Old }}$ & 19.100350099789003549065833483006 Trillion miles \\
\hline Old Equatorial radius & $R_{\text {EQUATOR-Old }}$ & 6,378.51495239773185755559473996188 Km \\
\hline Old Equatorial diameter & $D_{\text {earth-Equatorial }}^{\text {Old }}$ & $12,757.029904795463715111894799238 \mathrm{Km}$ \\
\hline Old Earth density & $E D_{\text {old }}$ & $5.5152740465837649209294596615615 \mathrm{gr} / \mathrm{cm}^{3}$ \\
\hline Old Volume percent of helium gas in dry atmosphere & $V P_{\text {Helium gas-dry atm. }}^{\text {Old }}$ & $5.2400000000000000000000000000001 \mathrm{e}-4$ \\
\hline Old Volume percent of xenon gas in dry atmosphere & $V P_{\text {Xenon gas-dry atm. }}^{\text {Old }}$ & 0.000009 Percent by volume \\
\hline Old Diameter of earth & $D_{\text {earth-Old }}$ & $12,349.648315313629613055429259477 \mathrm{Km}$ \\
\hline Old Radius of earth & $R_{\text {earth-Old }}$ & 6,174.8241576568148065277146297385 Km \\
\hline Old Earth's orbital inclination to sun's equator & $O I_{\text {to sun's equator-Old }}$ & $7.1902396858048243524900499244068^{\circ}$ \\
\hline Old Age of the earth & $\mathrm{AOE}_{\text {Old }}$ & 4.5501750498945017745329167415032 billion years \\
\hline Old strength limit of earth & $X_{C-\text {-arth oLD }}$ & $\begin{array}{l}0.64712157172243419172410449319661 \mathrm{MPa} \\
64.712157172243419172410449319661 \%\end{array}$ \\
\hline $\begin{array}{l}\text { Old gravity of earth or } \\
\text { standard gravity of earth }\end{array}$ & $g_{0-\text { old }}$ & $9.806649999999999999999999999999999 \mathrm{~m} / \mathrm{s}^{2} 9.80665 \mathrm{~m} / \mathrm{s}^{2}$ \\
\hline Old Speed of light in ether or so-called vacuo & $C_{\text {old }}$ & $299,792,458 \mathrm{~ms}^{-1}$ \\
\hline
\end{tabular}

${ }^{b}$ Old values established based on whole creation process of earth.

Table 4. Various features based on present strength limit number of earth and standard gravityc.

\begin{tabular}{|c|c|c|}
\hline Descriptions & Abbreviations & Adjusted values \\
\hline Present Thickness of homosphere & $T H_{\text {Now }}$ & $\begin{array}{l}94.6073047258079999999999999999995 \mathrm{~km} \\
9,460,730,472,580.79999999999999999997\end{array}$ \\
\hline $\begin{array}{l}\text { Present Distance value that light travel along one year } \\
\text { according to present standard gravity }\end{array}$ & Distance Light $r 1$ year $_{\text {Now }}$ & $9.460730472580800 \times 10^{12} \mathrm{~km}$ \\
\hline Present 1Parsec in Km & $1 \mathrm{PC}_{\text {in km-Now }}$ & 31.548418963802739825285687636571 Trillion kilometers \\
\hline Present 1 Parsec in miles & $1 \mathrm{PC}_{\text {in miles-Now }}$ & 19.596150459074984227984337103851 Trillion miles \\
\hline Present Equatorial radius & $R_{\text {EQUATOR-Now }}$ & $6,893.7349506352440108787641611296 \mathrm{~km}$ \\
\hline Present Equatorial diameter & $D_{\text {earth-Equatorial }}^{\text {Now }}$ & $13,262.217413231307031950753587346 \mathrm{~km}$ \\
\hline Present Volume percent of helium gas in dry atmosphere & $V P_{\text {Helium gas-dry atm }}^{\text {Now }}$ & $5.2735476347067444139118259313846 \mathrm{e}-4$ \\
\hline Present Volume percent of xenon gas in dry atmosphere & $V P_{\text {Xenon gas-dry atm }}^{\text {Now }}$ & $8.999999999999999999999999999999997 \mathrm{e}-6$ \\
\hline Present Earth density & $E D_{\text {Now }}$ & $5.1515426224147926418976043027263 \mathrm{gr} / \mathrm{cm}^{3}$ \\
\hline Present Diameter of earth & $D_{\text {earth-Now }}$ & $12,757.029904795463715111894799238 \mathrm{~km}$ \\
\hline Present Radius of earth & $R_{\text {earth-Now }}$ & $6,419.3516107173017702367833422539 \mathrm{~km}$ \\
\hline Earth's orbital inclination to sun's equator & $O I_{\text {to sun's equator-Now }}$ & $7.4749783200352580613423877140914^{\circ}$ \\
\hline $\begin{array}{l}\text { Accurate or exact age of the earth according to present } \\
\text { standard gravity at present time }\end{array}$ & Age of the earth Exact $_{\text {or }} \mathrm{AOE}_{\text {Exact }}$ & 4.7303652362904 billion years \\
\hline Present strength limit of the earth & $X_{C-\text { earth }}{ }_{\text {Exact }}$ & $\begin{array}{l}0.67274804880317322552081489426819 \mathrm{MPa} \\
0.6727480488031732255208148942682 \%\end{array}$ \\
\hline Present gravity of earth & $g_{0-\text { Now }}$ & $10.195000354006777301014825930477 \mathrm{~m} / \mathrm{s}^{2}$ \\
\hline
\end{tabular}

'Present values of strength limit number of earth, standard gravity and other present values work effectively and established in initial process of earth's creations. 
Table 5. Formulas for various important features by old and present strength limit number of earth ${ }^{\mathrm{d}}$.

\begin{tabular}{|c|c|c|}
\hline \multicolumn{3}{|c|}{ Calculation various important features by strength limit number of earth } \\
\hline Parameters & Formulas & Equation No. \\
\hline & $T H=\left(e^{X_{C-\text { Earth }}}-1\right) \times 100=\frac{\text { Distance }_{\text {Lightw } 1 \text { year }}}{10^{11}}$ & $(82)$ \\
\hline Thickness of homosphere & $T H=\frac{2 g_{0}}{p \times e^{e} \times 10^{8}}=\frac{2 g_{0} \times \text { A.D }}{p \times 10^{8}}$ & (83) \\
\hline \multirow{7}{*}{$\begin{array}{l}\text { Distance value that light travel } \\
\text { along one year }\end{array}$} & $T H=\frac{2 X_{C-\text { earth }}}{p \times 10^{8}}$ & $(84)$ \\
\hline & Distance $_{\text {Light } \sim 1 \text { year }}=\left(e^{X_{C \text {-Earath }}}-1\right) \times 10^{13}$ & $(85)$ \\
\hline & 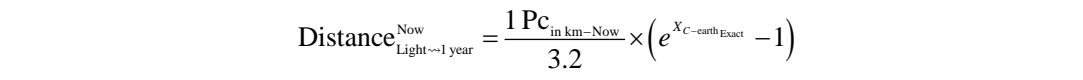 & (86) \\
\hline & 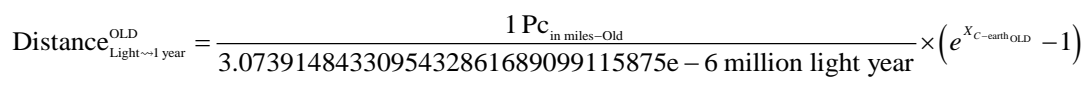 & (87) \\
\hline & Distance $_{\text {Lightw } 1 \text { year }}=T H \times 10^{11}$ & $(88)$ \\
\hline & Distance $_{\text {Light } w 1 \text { year }}=\frac{2 X_{C-\text { earth }}}{p \times 10^{-3}}$ & (89) \\
\hline & Distance $_{\text {Light } \rightarrow 1 \text { year }}=\frac{2 g_{0}}{p \times 10^{-3} \times e^{e}}=\frac{2 g_{0} \times \text { A.D }}{p \times 10^{-3}}$ & $(90)$ \\
\hline 1 Parsec in $\mathrm{Km}$ & $1 \mathrm{Pc}_{\text {in km }}=\frac{\text { Distance }_{\text {Light } \sim \sim \text { year }} \times 3.2}{e^{X_{C-\text { Earth }}}-1}$ & $(91)$ \\
\hline 1 Parsec in miles & $1 \mathrm{Pc}_{\text {in miles }}=e^{X_{C-\text {-atath }}} \times 10^{12}$ & (92) \\
\hline \multirow[t]{5}{*}{ Equatorial radius } & $R_{\text {EQUATOR }}=\left(\sec \left(X_{C \text {-Earth }}\right)-1\right) \times 10^{8}$ & $(93)$ \\
\hline & $E D_{\text {Now }}=X_{C \text {-earthoLD }}^{0.5665939193092108626257644267195 \times e} \times 10$ & $(94)$ \\
\hline & 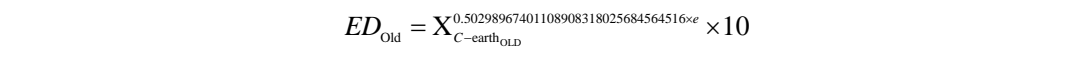 & $(95)$ \\
\hline & $E D_{\text {Now }}=X_{C-\text { earth }}^{0.6155906}$ & (96) \\
\hline & 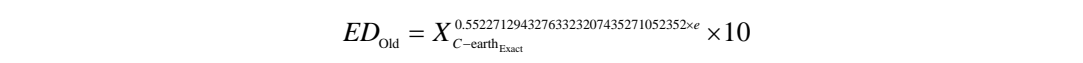 & $(97)$ \\
\hline \multirow[t]{3}{*}{ Earth density } & $E D_{\text {Now }}=\frac{g_{0-\text { Old }}}{100 X_{C \text {-earth oLD }}}+5=\frac{g_{0 \text {-Old }}+500 X_{C \text {-earth oLD }}}{100 X_{C \text {-earth oLD }}}$ & $(98)$ \\
\hline & $E D_{\text {Now }}=\frac{1+500 \mathrm{~A} . \mathrm{D}}{100 \mathrm{~A} \cdot \mathrm{D}}$ & (99) \\
\hline & 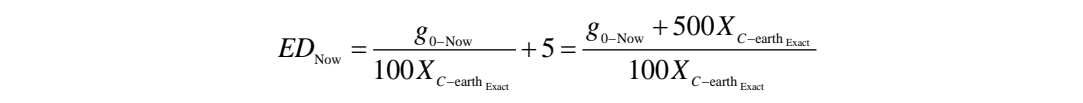 & $(100)$ \\
\hline Diameter of earth & $D_{\text {earth }}=\frac{10 X_{C-\text { Earth }}}{\text { Volumepercent of helium gas in dry atmosphere }}$ & $(101)$ \\
\hline $\begin{array}{l}\text { Volume percent of helium gas } \\
\text { in dry atmosphere }\end{array}$ & $V P_{\text {Helium gas-dry att. }}=\frac{10 X_{C-\text { Earth }}}{D_{\text {earth }}}=\frac{10 \times g_{0} \mathrm{~A} . \mathrm{D}}{D_{\text {earth }}}$ & $(102)$ \\
\hline $\begin{array}{l}\text { Volume percent of xenon gas } \\
\text { in dry atmosphere }\end{array}$ & $V P_{\text {Xenon gas-dry atm. }}=\frac{X_{C \text {-Earth }}}{\text { orbital inclination to sun's equator } \times 10^{4}}$ & $(103)$ \\
\hline $\begin{array}{l}\text { Earth's orbital inclination to } \\
\text { sun's equator }\end{array}$ & $O I_{\text {to sun's equator }}=\frac{X_{C-\text { Earth }}}{V P_{\text {Xenon gas-dry am. }} \times 10^{4}}$ & (104) \\
\hline
\end{tabular}

${ }^{\mathrm{d}}$ All general formulas are true based on old and present forms. 


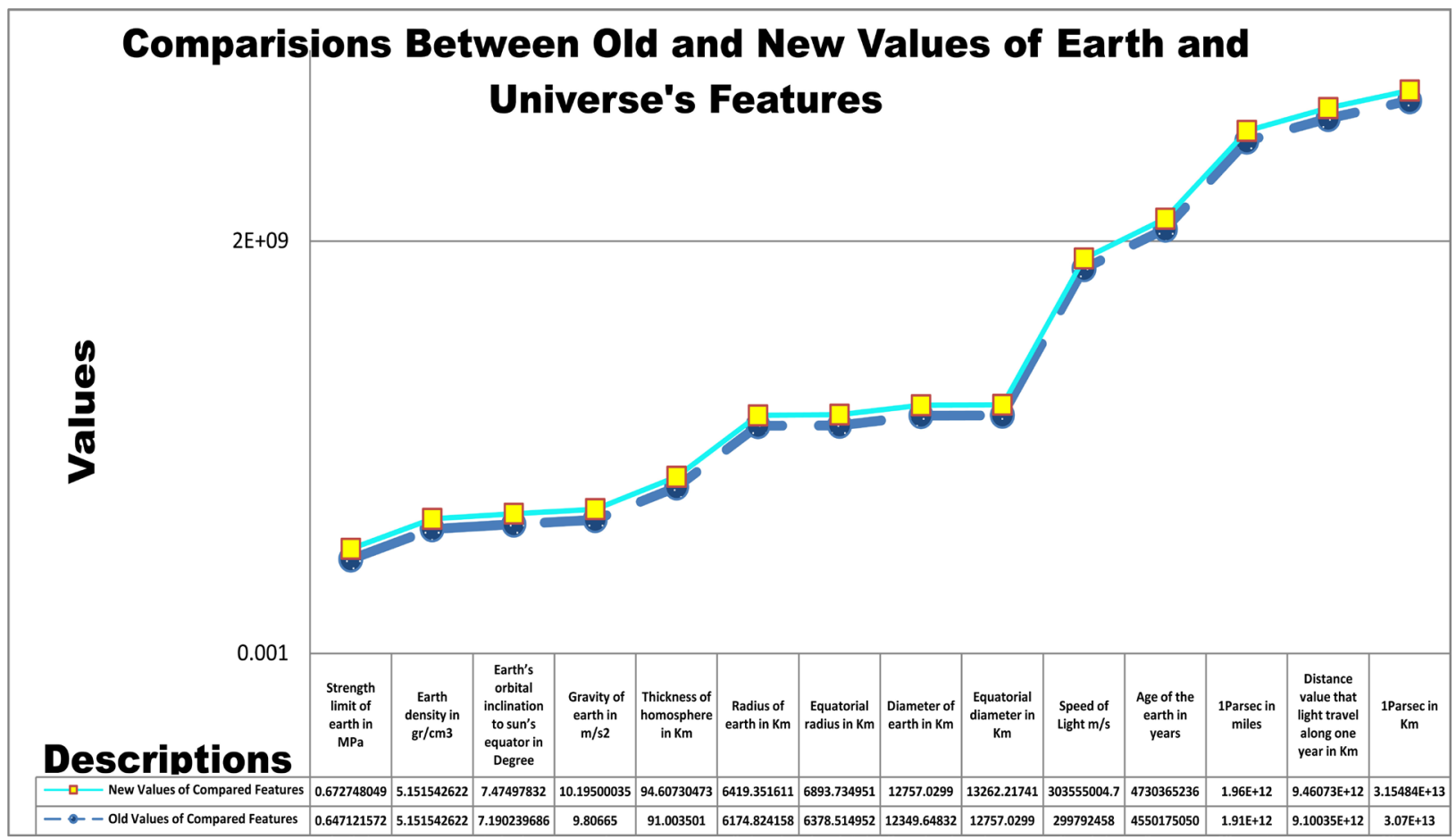

Figure 1. The comparison between some old and new values of earth and universe's specifications during life time of universe and earth.

\subsection{Some Applications of Strength Limit of Earth}

\subsubsection{Determination of Life Time Cycles of Earth through Napier's Definition in Series}

Napier's constant is a key number in existence description. Exponential process is a creating and mortality procedures. Above applications of " $\mathrm{e}$ " indicated the importance of this basis parameter that's an earth (existence) describer factor. Euler's constant in fact derived from earth characteristics (and overhand) that's play a principal role with earth's strength limit in response to different complicate and ambiguous questions. Exponential proceedings are one of the infinite ways to better cognition of cosmos or in the other words the supreme perception of universe.

Functions like $e^{x}$ and $e^{2 x}$ are linear independent on every interval. Euler's or Napier's definitions according to earth's features and series and parallel connection are given by:

$$
\begin{aligned}
e=\ln \left(\frac{g_{0}}{X_{C-\text { Earth }}}\right) & =\ln \left(\frac{G \times E}{R_{\text {Earth }}^{2} \times X_{C \text {-Earth }}}\right)=\ln \left(\frac{G \times E D \times V_{\text {Earth }}}{R_{\text {Earth }}^{2} \times X_{C-\text { Earth }}}\right)=\ln \left(\sum_{n=0}^{18} \frac{e^{n}}{n !}\right) \\
e & =\ln \left(\frac{g_{0 \text {-Old }}}{X_{C-\text {-earth OLD }}}\right)=\ln \left(\frac{g_{0 \text {-Now }}}{X_{C-\text { earth }_{\text {Exact }}}}\right) \ln \left(\frac{g_{0 \text {-Change }}}{X_{C-\text { earth Change }}}\right)
\end{aligned}
$$

where 18 is the number of earth's life time cycles or earth's changing cycles during life time. On the other hand 18 is the number of existence or universe (or a part of it) cycles or number of existence's life time cycles. 18 in fact is the age of the mother galaxy which contained Milky Way galaxy and earth in billion years. 
18 is the distance between cosmic objects, $\boldsymbol{g}_{\mathbf{0} \text {-Change }}$ is the changing gravity according to variations of earth's features and $\boldsymbol{X}_{\boldsymbol{C} \text {-earth }}$ Change $_{\text {is the changing }}$ strength limit of earth based on features of earth variations.

Above definition proves that existence like universe and Uno-verse undergo structural changing after 18 cycles. Any variations of earth's specifications if leads to unbalanced changing of the gravity, strength limit and other factors then key pivot numbers will change or will create another pivot numbers.

\subsubsection{Exact Perihelion Distance and Earth Melting Temperature Calculating by Old Strength Limit of Earth}

The ordinary Newtonian laws of gravitation seem too failed before, for the precession of the perihelion of planets. In this part shown here that alternatively a generalized approach via strength limit due to the inclusion of gravity can accounted well to determining the perihelion of planets like earth.

The point of the earth's orbit that is nearest to the sun have distance 0.98329134 AU or 147,098,290 km [71]. Earth's features such as earth melting temperature, strength limit of earth, Napier number, unit area density and etc. illustrates a formula that describing these relationships as the following form to identify the perihelion as:

$$
\begin{aligned}
& \text { Perihelion }_{\mathrm{OLD}}=\frac{X_{C-\text { earth }_{\mathrm{OLD}}} \times \text { Earth }_{. \mathrm{MT}} \times \mathrm{VDVR}_{X_{C-\text { Earth }}^{\text {Old }}}}{(\text { U.A.D }) \times e \times(\mathrm{U} . \mathrm{F}) \times(\mathrm{U} . \mathrm{T})} \\
& =\frac{g_{0 \text {-Old }} \times \text { A.D } \times \text { E.M.T } \times \mathrm{VDVR}_{X_{C-\text { Earth }}^{\text {Old }}}}{(\text { Unit Area Density }) \times \ln \left(\frac{G \times E D \times V_{\text {Earth }}}{R_{\text {Earth }}^{2} \times X_{C \text {-earth OLD }}}\right) \times(\text { U.F }) \times(\text { U.T })} \\
& \text { Perihelion }_{\mathrm{OLD}}=\frac{\left(g_{0-\mathrm{Old}} \times \text { A.D } \times 2065.1938510478206756666\right.}{1000 \times \ln \left(\frac{g_{0-\text { Old }}}{X_{C-\text { earth }_{\mathrm{OLD}}}}\right) \times 1 \times 1} \\
& =\frac{\left(X_{C-\text { earth OLD }} \times 2065.19385104782067566666425688\right) \times 2}{1000 \times \ln \left(\sum_{n=0}^{18} \frac{e^{n}}{n !}\right) \times 1 \times 1}
\end{aligned}
$$

$$
\text { Perihelion }_{\mathrm{OLD}}=0.98329134 \mathrm{AU}
$$

Balance of the units in Equation (107) is as follows:

$$
\begin{gathered}
\frac{\mathrm{MPa} \times{ }^{\circ} \mathrm{C} \times \mathrm{km}}{\frac{\mathrm{ton}}{\mathrm{m}^{2}} \times \frac{1}{\mathrm{~kg}} \times 1 \mathrm{kN} \times 1^{\circ} \mathrm{C}} \times \frac{1 \mathrm{AU}}{1 \mathrm{kM}}=\frac{\mathrm{M} \frac{\mathrm{N}}{\mathrm{m}^{2}} \times{ }^{\circ} \mathrm{C} \times \mathrm{km}}{1 \frac{\mathrm{Mgr}}{\mathrm{m}^{2}} \times \frac{1}{\mathrm{~kg}} \times 1 \mathrm{kN} \times 1^{\circ} \mathrm{C}} \times \frac{1 \mathrm{AU}}{1 \mathrm{kM}} \\
\frac{\mathrm{Mgr} \frac{\mathrm{km}}{\mathrm{m}^{2} \mathrm{~s}^{2}} \times{ }^{\circ} \mathrm{C} \times \mathrm{km}}{1 \frac{\mathrm{Mgr}}{\mathrm{m}^{2}} \times \frac{1}{\mathrm{~kg}} \times 1 \mathrm{kN} \times 1^{\circ} \mathrm{C}} \times \frac{1 \mathrm{AU}}{1 \mathrm{kM}}=\frac{\frac{\mathrm{km}}{\mathrm{s}^{2}} \times \mathrm{km}}{\frac{1}{\mathrm{~kg}} \times 1 \mathrm{kN}} \times \frac{1 \mathrm{AU}}{1 \mathrm{kM}} \\
=\frac{\frac{\mathrm{kg} \times \mathrm{km}}{\mathrm{s}^{2}} \times \mathrm{km}}{1 \mathrm{kN}} \times \frac{1 \mathrm{AU}}{1 \mathrm{kM}}=\frac{\mathrm{kN} \times \mathrm{km}}{1 \mathrm{kN}} \times \frac{1 \mathrm{AU}}{1 \mathrm{kM}}
\end{gathered}
$$


where Earth $_{\text {.MT }}$ or E.M.T is earth melting temperature which is the widespread contiguous temperature for melting all elements of earth's creation, U.F is the unit force $=$ Unit impressive force on every gravitational particle $=$ Total impressive forces involve in life time of gravitational particles (life time of gravitational particle's process) $=$ Effecting force by gravitational particles on area $=$ Effecting forces on gravitational particles by area $=1 \mathrm{kN}$ force in a large surrounding area $=$ Total Involving forces in life time of gravitational particles (life time of gravitational particle's process), U.T is the unit temperature and equal to 1 degree Celsius, $\mathbf{V D V R}_{\boldsymbol{X}_{\text {C-Earth }}}^{\text {Old }}$ is variations distance value range of earth's strength limit in 10 billion year according to old standard gravity and equal to 2 $\mathrm{km}$, U.A.D is the unit area density and equal to $1000 \mathrm{Mgr} / \mathrm{m}^{2}$ or $1000 \mathrm{ton} / \mathrm{m}^{2}$ or $1 \mathrm{Ggr} / \mathrm{m}^{2}$ and Perihelion $_{\text {OLD }}$ is the perihelion distance according to old strength limit of earth.

Coefficient 2 or variations distance value range of earth's strength limit in 10 billion year according to old standard gravity is calculated in the following form which derives from different methods like earth features as:

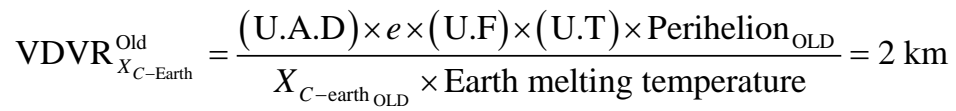

Another way to calculate this factor is described by Weil-Petersson volumes, symplectic geometry, intersection theory and volumes of moduli spaces of bordered Riemann surfaces as this form:

$\operatorname{VDVR}_{X_{C \text {-Earh }}}^{\text {Old }}=\sqrt[|\alpha|]{\frac{1}{C_{g} \times E|\alpha| !(3 g-3+n-|\alpha|)} \times \int_{\bar{M}_{g, n}} \psi_{1}^{\alpha 1} \cdots \psi_{n}^{\alpha n} \cdot \omega^{3 g-3+n-|\alpha|}}=2$

where $\overline{\boldsymbol{M}}_{g, n}$ is Deligne-Mumford compactification of the moduli space (of hyperbolic Riemann surfaces) of genus $g$ curves with $\mathrm{n}$ distinct marked points as a function of $\boldsymbol{g}$ and $\boldsymbol{n}, \psi_{i}$ is the first Chern class of the i-th tautological line bundle, $\omega$ is the Weil-Petersson symplectic form, the exponent $\alpha=\left(\alpha_{1}, \cdots, \alpha_{n}\right)$ ranges over elements in $(Z \geqslant 0)^{n}$ and $\alpha !=\prod_{i=1}^{n} \alpha_{i}$, and $|\alpha|=\sum_{i=1}^{n} \alpha_{i} \quad$ [72].

Then Euler's constant in accordance to perihelion, earth melting temperature, variations distance value range of earth's strength limit in 10 billion year according to old standard gravity, Weil-Petersson volumes, symplectic geometry, intersection theory and Riemann surfaces is equal to:

$$
\begin{aligned}
& e=\ln \left(\frac{g_{0}}{X_{C-\text { Earth }}}\right)=\ln \left(\sum_{n=0}^{18} \frac{e^{n}}{n !}\right) \\
& =|\alpha| \frac{1}{\frac{C_{g} \times E|\alpha| !(3 g-3+n-|\alpha|)}{C_{M_{g, n}}} \psi_{1}^{\alpha 1} \cdots \psi_{n}^{\alpha n} \cdot \omega^{3 g-3+n-|\alpha|}} \\
& \times \frac{\text { Earth melting temperature } \times X_{C \text {-Earth }}}{(\text { U.A.D }) \times(\text { U.F }) \times(\text { U.T }) \times \text { perihelion }}
\end{aligned}
$$

\subsubsection{Adjusting Earth's Rotation Parameters}

5.2.3.1. Conventional Duration of the Sidereal Day

Earth's rotation period relative to the processing or moving mean vernal equi- 
nox, misnamed its sidereal day, is $86,164.09053083288$ seconds of mean solar time (UT1) $23 \mathrm{~h} 56 \mathrm{~m} 4.09053083288 \mathrm{~s}$ [73]. Conventional duration of the sidereal day based on Aoki et al., in 1982 is equal to $86,164.09053083288 \mathrm{~s}$ or 23.934469591898022222222222222222 hour. Sidereal day based on strength limit of earth and surface density adjusted as following forms:

$$
\begin{aligned}
\text { Earth }_{\text {SRD-OLD }} & =\frac{\left(U_{\text {A.D }}\right)^{2} \times U_{\text {SRD-Velocity }}}{X_{\text {C-earth OLD }} \times \text { A.D }}=\frac{\left(U_{\text {A.D }}\right)^{2} \times U_{\text {SRD-Velocity }}}{g_{0-\text { Old }} \times(\text { Area Density })^{2}} \\
& =23.93446959189802222222222222221 \mathrm{~h} \\
\text { Earth }_{\text {SRD-Now }} & =\frac{\left(U_{\text {A.D }}\right)^{2} \times U_{\text {SRD-Velocity }}}{X_{C-\text { earth }_{\text {Exact }}} \times \text { A.D }}=\frac{\left(U_{\text {A.D }}\right)^{2} \times U_{\text {SRD-Velocity }}}{g_{0-\text { Now }} \times(\text { Area Density })^{2}} \\
& =23.022752140576405075042839103192 \mathrm{~h}
\end{aligned}
$$

where strength limit of earth is the stress-pressure bearing capability limit or enduring elasticity coefficient (EEC) or resistive elasticity factor (REF) of earth, U.A.D or $\boldsymbol{U}_{\text {A.D }}$ is the unit area density and equal to $1 \mathrm{Ggr} / \mathrm{m}^{2}, \boldsymbol{U}_{\text {SRD-Velocity }}$ is the unit sidereal day velocity and equal to 1.0220564573745930299341129304479

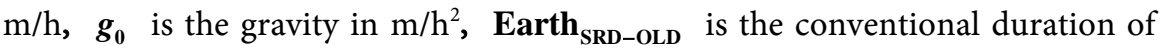
the sidereal day of earth according to old enduring elasticity coefficient in hours, Earth $_{\text {SRD-Now }}$ is the conventional duration of the sidereal day for earth according to present resistive elasticity factor in hours and A.D is area or surface density and equal to $0.06598803584531253707679018759685 \mathrm{Ggr} / \mathrm{m}^{2}$.

It's important to mentioned that strength limit of earth has direct proportion to gravity and on the other hand has reverse proportion to sidereal day value. Therefore value of sidereal day changed by variation of standard gravity and strength limit of earth.

The adjusted sidereal day in 4.73 billion years at the beginning time of the earth's creation is shorter than the calculated sidereal day value in 4.55 billion years at whole creation time of earth.

\subsubsection{Earth Obliquity to Orbit}

Earth obliquity to orbit according to old and present strength limit, gravity and area density are given as:

$$
\begin{aligned}
& \text { Earth }_{\text {OTO-Old }}=\frac{\left(U_{\text {A.D }}\right)^{2} \times U_{\text {Gravity }}}{X_{\text {C-earth OLD }} \times \text { Earth's Area Density }}=\frac{\left(U_{\text {A.D }}\right)^{2} \times U_{\text {Gravity }}}{g_{0-\text { Old }} \times(\text { A.D })^{2}} \\
& =23.417952520333052827410949589963^{\circ} \\
& \text { Earth }_{\text {OTO-Now }}=\frac{\left(U_{\text {A.D }}\right)^{2} \times U_{\text {Gravity }}}{X_{\text {C-earth Exact }} \times \text { A.D }}=\frac{\left(U_{\text {A.D }}\right)^{2} \times U_{\text {Gravity }}}{g_{0-\text { Now }} \times(\text { Area Density })^{2}} \\
& =22.525910358920961312091602878378^{\circ}
\end{aligned}
$$

where strength limit of earth is the stress-pressure bearing capability limit or enduring elasticity coefficient (EEC) or resistive elasticity factor (REF) of earth, U.A.D or $\boldsymbol{U}_{\mathrm{A} . \mathrm{D}}$ is the unit area density and equal to $1 \mathrm{Ggr} / \mathrm{m}^{2}, \boldsymbol{U}_{\text {Gravity }}$ is the unit gravity and equal to $1 \mathrm{~m} / \mathrm{h}^{2}, \boldsymbol{g}_{0}$ is the gravity in $\mathrm{m} / \mathrm{h}^{2}$, Earth ото-old is 
the obliquity to orbit for earth according to old enduring elasticity coefficient and Earth $_{\text {ото-Now }}$ is the obliquity to orbit for earth according to present resistive elasticity factor.

\subsubsection{Main Apparent Motion of Celestial Bodies in the Earth's Sky}

Apart from meteors within the atmosphere and low-orbiting satellites, the main apparent motion of celestial bodies in the Earth's sky is to the west at a rate of $15^{\circ} / \mathrm{h}=15^{\prime} / \mathrm{min}$. For bodies near the celestial equator, this is equivalent to an apparent diameter of the Sun or Moon every two minutes; from the planet's surface, the apparent sizes of the Sun and the Moon are approximately the same [61] [74].

The adjusted main apparent motion of celestial bodies in the Earth's sky is to the west at a rate of $15.15426224147926418976043027263^{\circ} / \mathrm{h}=\mathrm{e}^{\mathrm{e}} / \mathrm{min}$.

\subsubsection{Cosmic Speed Measurement or Measurement of the Universe's Expansion Rate}

Scientists have known about this acceleration since the late $1990 \mathrm{~s}$, but whatever is causing it-dubbed dark energy-remains a mystery. Now the latest measurement of how fast the cosmos is growing thickens the plot further: The universe appears to be ballooning more quickly than it should be, even after accounting for the accelerating expansion caused by dark energy [75].

The newest unprecedentedly precise value measurement for the Hubble constant comes out to 45.5 miles (73.2 kilometers) per second per mega parsec which puts the expansion rate of universe at 73.02 , plus or minus 1.79 , kilometers per second per mega parsec (One mega parsec is equivalent to 3.26 million light-years), meaning that for each mega parsec you go out, space is receding about 73 kilometers a second faster [75] [76].

The European Space Agency's Planck Satellite defined the expansion rate of the universe at 67.3, plus or minus 0.7 , kilometers per second per mega parsec according to the best observations to date of the cosmic microwave background (CMB) [Adopted from [75]].

Another way to calculate the universe expansion rate came from the exact overall age of the earth. This measure in accordance to the age of the earth from the beginning time of earth's creation process till now obtained by:

$$
\begin{aligned}
& \text { UNIVERSE }_{\text {Expansion Rate }}^{\text {Exact Age Thrh }} \\
& =\left(\operatorname{AOE}_{\text {Exact }}-n\right) * \mathrm{ER}_{\mathrm{UNIT}}=73.03652362904 \mathrm{~km} / \mathrm{sMegaparsec} \\
& \text { UNIVERSE }_{\text {Expansion Rate }}^{\text {Exact Age of The Earth }} \\
& =45.392494486662523306401491609695 \mathrm{Mile} / \mathrm{sMegaparsec}
\end{aligned}
$$

where $\boldsymbol{n}$ is the earth's life cycles which to be traversed,

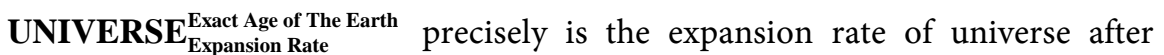
4.7304 billion years (exact age of the earth) from the beginning time of the earth's creation process, $\mathbf{A O E}_{\text {Exact }}$ is the exact age of the earth according to present standard gravity and equal to 4.7303652362904 billion years and 
$\mathbf{E R}_{\mathrm{UNIT}}$ is the Unit expansion rate range in 1 billion year and equal to 100 $\mathrm{km} /$ Second Megaparsec billion year.

Resistive elasticity factor (REF) of earth or strength limit has direct proportion to universe expansion rate. Expansion rate of universe based on strength limit of earth in initial time situation of earth's creation process and using involved gravity obtained by:

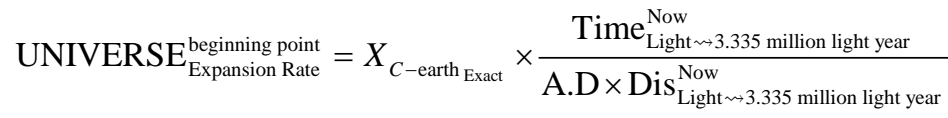

$$
\begin{aligned}
& \text { UNIVERSE } E_{\text {Expansion Rate }}^{\text {beginning point }}=g_{0-\text { Now }} \times \frac{\text { Time }_{\text {Light } \rightsquigarrow 3.335 \text { million light year }}^{\text {Now }}}{\text { Dis }_{\text {Light } \rightsquigarrow 3.335 \text { million light year }}^{\text {Now }}} \\
& \text { UNIVERSE } E_{\text {Expansion Rate }}^{\text {beginning point }} \\
& =67.27480488031732255208148942682 \mathrm{~km} / \mathrm{sMegaparsec}
\end{aligned}
$$

In fact this rate is the expansion in the beginning point of the earth's creation process and during creation process till before whole creation situation. Hence, the cosmic expansion rate at whole creation state is written as:

$$
\begin{gathered}
\text { UNIVERSE }_{\text {Expansion Rate }}^{\text {Whole Creation }}=X_{C-\text { earth OLD }} \times \frac{\text { Time }_{\text {Light } \rightsquigarrow 3.4 \text { million light year }}^{\text {OLD }}}{\mathrm{A} . \mathrm{D} \times \mathrm{Dis}_{\text {Light } \rightsquigarrow 3.4 \text { million light year }}^{\text {OLD }}} \\
\text { UNIVERSE }_{\text {Expansion Rate }}^{\text {Whole Creation }}=g_{0-\text { Old }} \times \frac{\text { Time }_{\text {Light } \rightsquigarrow 3.4 \text { million light year }}^{\text {OLD }}}{\operatorname{Dis}_{\text {Light } \rightsquigarrow 3.4 \text { million light year }}^{\text {OLD }}}
\end{gathered}
$$

where $\boldsymbol{X}_{C-\text { earth }_{\text {Exact }}}$ is present strength limit of earth in (dimensionless or percent), $\boldsymbol{X}_{C \text {-earth oLd }}$ is old strength limit of earth (dimensionless or percent), Time Light $\sim 3.4$ million light year $_{\text {OLD }}$ is time needed that light travel about 3.4 million light year and equal to 6598.803584531253707679018759685 second,

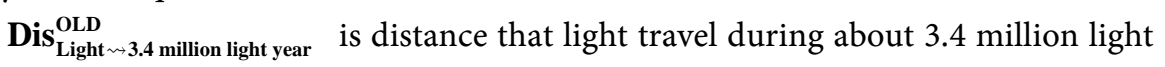
year (3.3777984468760313238883943075476 million light years) and equal to old value of 1 Mega parsec ( 1 parsec $=3.3777984468760313238883943075476$ light year), Time Light $\sim 3.335$ million light year $_{\text {Now }}$ time needed that light travel about 3.335 million light year and equal to 6598.803584531253707679018759685 second, Dis $_{\text {Light } ~}^{\text {Now.335 million light year }}$ is distance that light travel during about 3.335 million light year (3.3346705156897490768906328666323 million light years) and equal to 1 Mega parsec, A.D is the area density and equal to $65.98803584531253707679018759685 \mathrm{Mgr} / \mathrm{m}^{2}, \boldsymbol{X}_{C-\text { earth }_{\text {Exact }}}$ is the present strength limit of earth and equal to $67.27480488031732255208148942682 \mathrm{MPa}$ and $\boldsymbol{X}_{\text {C-earth }}$ old is the old strength limit of earth and equal to 64.712157172243419172410449319661 MPa.

When present exact strength limit of earth role in self dimensionless form in fact express the real expansion rate with $1 \mathrm{~km} / \mathrm{s}$ Megaparsec expansion rate unit in presence of 100 percent of total forces involved in existence. The expansion rate of universe in accordance to the beginning time of the earth creation process is $67.27480488031732255208148942682 \mathrm{~km} / \mathrm{s}$ Megaparsec: 


$$
\begin{aligned}
& \text { UNIVERSE }_{\text {Expansion Rate }}^{\text {beginning poit }}=\left(X_{C-\text { earth Exact }} \%\right) \times\left(U_{E R}=100 \frac{\mathrm{km} / \mathrm{sMegaparsec}}{1 \mathrm{MPa}}\right) \\
& \text { UNIVERSE }_{\text {Expansion Rate }^{\text {begining point }}}=\left(X_{C-\text { earth }_{\text {Exact }}} \%\right) \times\left(100 \%=1 \frac{\mathrm{km} / \mathrm{sMegaparsec}}{1 \%}\right) \\
& \text { UNIVERSE } E_{\text {Expansion Rate }}^{\text {begining point }} \\
& =67.27480488031732255208148942682 \mathrm{~km} / \mathrm{sMegaparsec}
\end{aligned}
$$

where $\boldsymbol{U}_{\boldsymbol{E} \boldsymbol{R}}$ is the unit universe expansion rate in accordance to $1 \mathrm{MPa}$ strength limit.

Expansion rate of universe in whole creation process time point of earth calculated as:

$$
\begin{aligned}
& \text { UNIVERSE }_{\text {Expansion Rate }}^{\text {Whol Cration }}=X_{C \text {-earth oLD }} \\
& =64.712157172243419172410449319661 \mathrm{~km} / \mathrm{sMegaparsec}
\end{aligned}
$$

The universe is expanding according to exact age of the earth 5 to 9 percent (exactly $3.9601 \%-8.5645 \%$ ) faster than expansion rate value according to present strength limit of earth. Universe expansion rate was

$64.712157172243419172410449319661 \mathrm{~km} / \mathrm{s}$ Megaparsec when earth put in whole creation process time.

\subsubsection{Discovery of Other Fundamental Forces of Nature}

Discoveries of strength limit of earth, accurate age of the earth, gravity variations, earth's area density, strength variations coefficient from age viewpoint and other factors helps to found other fundamental forces of nature. How these will happen?

Previously definitions of age of the earth came from the relations between strength limit of earth and strength variations coefficient spotted an anomaly in radioactive decay value of gravitational particles close to ${ }^{87} \mathrm{Rb}$ that could be the signature of other unknown fundamental forces of nature.

Variations in decay factor especially in decay factor of gravitational particles can find out unknown important fundamental elements and particles. Strength limit of earth on the other hand is a fundamental equalizer forces. Mentioned outlook can be figured by:

$$
\begin{aligned}
& \text { Gravity } \times \text { Area Density }=\text { Age of the earth } \times p \\
& =\text { Age of the earth } \times \text { D.C } \times(\text { MEARS }) \\
& \text { Strength Limit of Earth } \\
& =\text { Age of the earth } \times \text { Decay Constatnt } \times(\text { MEARS }) \\
& \quad X_{C \text {-earth }}=\text { AOE } \times \text { D.C } \times(\text { MEARS })
\end{aligned}
$$

where [41] $\boldsymbol{p}$ is strength variations coefficient from age viewpoint (strength variations coefficient in $10^{10}$ year or strength variations coefficient with regard to overall age of the earth and equal to $p=1.4221904973467736128057926319335 \times 10^{-10}$ in $\mathrm{MPa} / \mathrm{Year}=\mathrm{MN} /\left(\mathrm{m}^{2}\right.$ Year $)=\mathrm{Ggr} /\left(\right.$ Year $\left.\mathrm{m} \mathrm{s}^{2}\right)$, D.C is the decay constant of gravitational particles. Currently exact value for the decay constant of ${ }^{87} \mathrm{Rb}$ is 
$1.4221904973467736128057926319335 \times 10^{-11} \mathrm{yr}^{-1}$. This value in fact is the constancy of decay rate quantity of the mother isotope of gravitational elements and particle's atoms and MEARS is the minimal effective action and reaction unit of strength and equal to $10 \mathrm{MPa}$.

Strength variations coefficient computed as:

$$
\begin{aligned}
& p=\frac{X_{C-\text { Earth,Old }}}{\mathrm{AOE}_{\text {Old }}}=\frac{g_{0-\text { Old }} \times \frac{1}{e^{e}}}{\mathrm{AOE}_{\text {Old }}}=\frac{g_{0-\text { Old }} \times \mathrm{A} . \mathrm{D}}{\mathrm{AOE}_{\text {Old }}} \\
& p=\frac{X_{C-\text { Earth,Now }}}{\mathrm{AOE}_{\text {Exact }}}=\frac{g_{0-\text { Now }} \times \frac{1}{e^{e}}}{\mathrm{AOE}_{\text {Exact }}}=\frac{g_{0-\text { Now }} \times \mathrm{A} . \mathrm{D}}{\mathrm{AOE}_{\text {Exact }}}
\end{aligned}
$$

\subsubsection{Specifying Accurate Present Total Number of Seconds in One Year} and Precise Distance of Earth from the Sun and Adjusting Units

Distance from the sun according to previous computation is introduced as 149.6 $\mathrm{Km}$ [Adopted from [61]]. The relation between present total number of seconds in a defined year and distance of earth from the sun appears by exact age of the earth and occurrence time range of principle great structural changes in universe that calculates based on strength limit number of earth. According to the last changes in features of earth and universe mentioned parameters calculated as:

Present total number of seconds in a defined year $=\frac{\text { Distance }_{\text {from sun }}^{\text {Now }} \times \text { RMCS }}{\mathrm{AOE}_{\text {Exact }} \times \text { V.earth }}$

$$
\begin{aligned}
\text { PTNS }_{\text {Now }} & =\frac{149597869.9456460177916343695247 \mathrm{~km} \times 10^{10} \text { year }}{4.7303652362904 \times 10^{9} \text { year } \times 10 \mathrm{~km} / \mathrm{s}} \\
& =31625014.660170759282639202329243 \mathrm{~s}
\end{aligned}
$$

where Distance from sun $_{\text {Now }}$ distance of earth from the sun and equal to 149.5978699456460177916343695247 $\times 10^{6} \mathrm{~km}$, RMCS or PGCOTR is the occurrence time range unit of principle great structural changes in universe or time range of great structural variations occurrence possibility or revolutionary mutation changes span or principle great structural revolutionary mutation changes

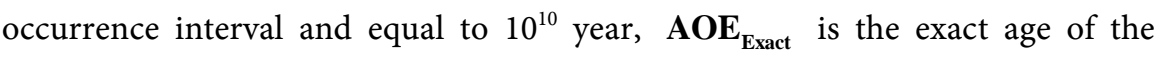
earth according to present standard gravity in years and equal to

$4.7303652362904 \times 10^{9}$ years, PTNS is present total number of seconds in a defined year, PTNS $_{\text {Now }}$ is the total number of seconds factor for the beginning time of earth creation and is equal to 8784.7262944918775785108895359008 hour or 366.03026227049489910462039732921 day and V.earth IOc is the initial orbital changes velocity of earth with respect to sun, contained galaxy and universe conditions or the rate per seconds of time at which earth body moves in its specified orbital direction and equal to $10 \mathrm{~km} / \mathrm{s}$.

Present total number of seconds for a defined year use to adjusting units values like as determining the exact astronomical unit and perihelion value. Exact astronomical unit as a derived constant according to Equation (131) is equal to: $1 \mathrm{AU}=149,597,869.9456460177916343695247 \mathrm{~km}$. 
The exact perihelion distance is equal to $0.98329134000000000000000000000002 \mathrm{AU}$.

The initial orbital changes velocity of earth with respect to sun based on strength limit and earth density in old and present forms denoted by:

$$
\begin{aligned}
& \text { V.earth }_{\mathrm{IOC}}=\frac{\mathrm{ED}_{\mathrm{Now}}}{X_{C-\text { earth }}^{0.5605 \mathrm{LD}}} \\
& =\frac{\mathrm{ED}_{\mathrm{Old}}}{X_{C-\text { earth }}^{0.502969701108908318025684564516 \times e}}=10 \mathrm{~km} / \mathrm{s} \\
& \text { V.earth }_{\mathrm{IOC}}=\frac{\mathrm{ED}_{\text {Now }}}{X_{C-\text { earth }_{\text {Exact }}}^{0.615591529611795463607929 \times e}} \\
& =\frac{\mathrm{ED}_{\text {Old }}}{X_{C-\text { earth }}^{0.552274272763323207435271052352 \times e}}=10 \mathrm{~km} / \mathrm{s}
\end{aligned}
$$

\subsubsection{Recognizing the Particles Specifications Varieties}

Another application of precise defining of the total number of seconds for any defined year is showing very small but determinant changes in specifications of particles at different time particular main internal recycling time of neutrons. Main internal recycling time of neutron at the beginning time of earth creation is higher than its value at the whole creation process which displays in below equation:

$$
\begin{aligned}
& \text { ELTGP }_{\text {Now }}=\text { PTNS }_{\text {Now }} \times \mathrm{TF}_{\text {time }} \\
& =\frac{\text { Distance }_{\text {from sun }}^{\text {Now }} \times \mathrm{RMCS}}{\mathrm{AOE}_{\text {Exact }} \times \mathrm{V} \text {.earth }} \times 0.1 \mathrm{~s} / \mathrm{h}
\end{aligned}
$$

where ELTGP is the exact life time of gravitational particles according to unit of time or main internal recycling time of neutron that is equal to 878.41046115788314777747042900908 Sec, ELTGP $_{\text {Now }}$ is the exact life time of gravitational particles in the beginning time of the earth's creation process and equal to $878.47262944918775785108895359008 \mathrm{Sec} . \mathbf{T F}_{\text {time }}$ is the transformer factor with regard to times variations for one hour and equal to $0.1 \mathrm{~s} / \mathrm{h}$ and PTNS $_{\text {Now }}$ is the total number of seconds factor for the beginning time of earth creation in hour.

\subsubsection{Variable Speed of Light Phenomena; Are Speed of Light Change Phenomenally?}

Door needs to have a constant pivot for rotating in constant or dynamic positions. Physicists continue to ask today like as decades ago. Why constants are existing? Why is it a constant? How to be constant? Is it constant everywhere in the universe?

No theory could explain existence of constant numbers in science and their philosophies until now. These constant sounds universal and they emerge to be unchanging. The validation of constants in sciences be accomplished through observation and experiment ways, but not contemplate by theory. Measurements of constants on earth are useful and a good way to prove and determine the 
truth and accuracy of theories that using constants also be assure which science behind them stands on firm and true ground is measuring them. Discriminant error from these measurements can be illustrative as a beneficial concern.

Strength limit number of earth demonstrates constant numbers may be constant instantly in an era based on defined conditions. Measuring and validating the changes of speed of light based on strength limit as number that can be free of the burden of units is useful to reach to the destiny which are speed of light variable? Light specifications like its velocity are dependent to the other characteristics of earth such as total number of days in the same year which light traveled by mentioned specified peculiarities. Speed of light has direct proportion to distance that it travel during one year and total number of seconds in that same year. On the other hand for this distance, there is indirectly proportion to total number of days in that specified year and total number of hours in days of that same year. These relevancies based on previous knowledge are given by:

$$
\begin{gathered}
\text { Distance }_{\mathrm{Light} w 1 \text { year }}^{\mathrm{PN}} / \text { Speed of light }_{\mathrm{PN}}=\text { Time }_{\mathrm{Light} \sim 1 \text { year }}^{\mathrm{PN}} \\
\text { Distance }_{\mathrm{Light} \sim 1 \text { year }}^{\mathrm{PN}} / \text { Speed of light }_{\mathrm{PN}} \\
=\text { Total number of seconds in that year }
\end{gathered}
$$

where Distance $\mathbf{L i g h t ~} \sim 1$ year $_{\text {PN }}$ is value of distance that light travel in one year based on previous knowledge and equal to 9,460,730,472,000,000 Km, Speed of light pN $_{\mathbf{P N}}$ is the speed of light based on previous knowledge and equal to $299,792,458 \mathrm{Km} / \mathrm{s}$ and $\mathbf{T i m e}_{\mathrm{Light} \sim 1}^{\mathrm{PN}}$ year is the time that light needs to travel during one year furthermore is the total number of seconds in that year and equal to

$31,557,599.998062659735089132896065 \mathrm{sec}$ or 8765.9999994618499264136480266847 hour or 365.2499999775770802672353344452 day (if one day is equal to 24 hours in this equation).

Earth density quantity at whole creation point is bigger than its value at the beginning time of the earth creation. Mass to volume ratio of the earth at starting creation procedures is smaller than whole creation statement. Hence, mass of the earth in the starting time of the earth's creation has smaller amounts rather than the whole creation process. For that reasons according to the relativity of mass and speed of light and energy momentum, the light velocity at the earth creation processes' starting time has much more value than the whole creation point.

Light travel higher distance during one year in lower time at the beginning point of the earth creation. Present total number of seconds for a defined year use to assay the variety of speed of light in different times of changes in general, old and present forms as follows:

$$
\begin{gathered}
\left(\text { Distance }_{\text {Light } \sim 1 \text { year }} / \text { Total number of seconds in that year }\right)=\text { Speed of light } \\
\left(\text { Distance }_{\text {Light } \sim 1 \text { year }}^{\text {Now }} / \text { Present total number of seconds }\right)=\text { Speed of light } \\
\text { Now }_{\text {Distance }}^{\text {Old }}
\end{gathered}
$$




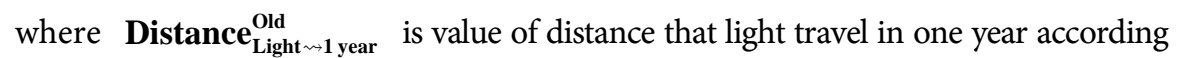
to old standard gravity and equal to $9.1003500997890035490658334830064 \times 10^{12}$ $\mathrm{km}$, Distance $\mathbf{L}_{\text {Light } \sim 1 \text { year }}^{\text {Now }}$ is value of distance that light travel in one year according to present standard gravity and equal to $9.460730472580800 \times 10^{12} \mathrm{~km}$ [41], Present total number of seconds is the total number of seconds at the beginning point of the earth's creation proceeding, Old total number of seconds is the total number of seconds at whole creation time of earth, Speed of light ${ }_{\text {Now }}$ is the speed of light at the starting point of earth's creation and

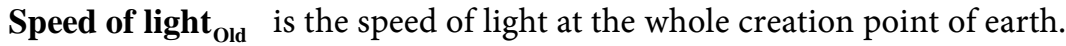

Follow relation is feasible based on the relevancies between the speed of light and electrical and magnetic field constants:

$$
C^{2}=\frac{1}{\varepsilon_{0} \times \mu_{0}}
$$

According to the correlation between speed of light and the electrical and magnetic field specifications conclude that any changes in speed of light variations leads to change the conditions of electrical and magnetic fields proportionally. Used factors in speed of light determinations in various eras are shown in Table 6.

Table 6. Speed of light variations determinant parameters.

\begin{tabular}{|c|c|c|}
\hline Distance that light travel in 1 year $(\mathrm{km})$ & Speed of light $(\mathrm{km} / \mathrm{s})$ & Total number of seconds in 1 year (Second) \\
\hline $\begin{array}{l}\text { Distance } \\
\text { Light } \sim 1 \text { year } \\
=9.460730472000000 \times 10^{12} \mathrm{~km}\end{array}$ & $\begin{array}{l}\text { Speed of light }_{\mathrm{PN}} \\
=299792.458 \mathrm{~km} / \mathrm{s}\end{array}$ & $\begin{array}{c}31,557,599.998062659735089132896065 \mathrm{Sec}= \\
365.2499999775770802672353344452 \text { Days }\end{array}$ \\
\hline $\begin{array}{l}\text { Distance }_{\text {Light } w 1 \text { year }}^{\text {Old }} \\
=91.003500997890035490658334830064 \times 10^{12} \mathrm{~km}\end{array}$ & $299,792.458 \mathrm{~km} / \mathrm{s}$ & $30,355,500.470225316839244279731034 \mathrm{~s}$ \\
\hline $\begin{array}{l}\text { Distance }_{\text {Light } w 1 \text { year }}^{\text {Now }} \\
9.460730472580800 \times 10^{12} \mathrm{~km}\end{array}$ & $\begin{array}{l}\text { Speed of light }_{\text {Now }} \\
=299792.45801840444140351787415343 \mathrm{~km} / \mathrm{s}\end{array}$ & $\begin{array}{c}31,557,599.998062659735089132896065 \mathrm{Sec}= \\
365.2499999775770802672353344452 \text { Days }\end{array}$ \\
\hline
\end{tabular}

If light at the beginning point of the earth creation travel with same speed like as the whole creation time therefore total number of seconds in that year face with higher amounts in value also for distance that light travel during one year too. Variations in total number of seconds in one year and distance that light travel during one year based on same speed of light for the begging time of the earth creation process era is shown in Table 7.

Table 7. Other factors changes due to same speed of light.

\begin{tabular}{clc}
\hline Distance that light travel in 1 year $(\mathrm{km})$ & Speed of light $(\mathrm{km} / \mathrm{s})$ & Total number of seconds in 1 year (Second) \\
\hline Distance $_{\text {Ligh } \sim 1 \text { year }}^{\text {Now }}$ & Speed of light ${ }_{\text {old }}$ & $31,557,600$ Sec \\
$=9.460730472580800 \times 10^{12} \mathrm{~km}$ & $=299792.458 \mathrm{~km} / \mathrm{s}$ & \\
\hline
\end{tabular}

\subsubsection{New Definitions of Euler's Constant or Napier's Constant}

Different definitions of Euler's constant based on Equation (1) up to Equation (139) shown in Table 8. It's necessary query one question that's Does this constant varies with time lapse? According to formulas that shown in Table 8: Does 
"e" varies by variations of strength limit of earth, standard gravity, thickness of homosphere, distance that light travel during one year, age of the earth, earth density, earth melting temperature, volume of earth, radius of earth and other related factors?

If standard gravity, strength limit of earth, and other earth's features (mass, volume, radius, density and etc.) change with non-constant rate, then "e" will change and convert to a new fundamental equalizer factor.

Table 8. Different definitions of Euler number.

\begin{tabular}{|c|c|}
\hline $\begin{array}{l}\text { Different Equations for Calculating Napier's Number } \\
\text { from Earth's Features }\end{array}$ & Equations Number \\
\hline$e=\frac{\ln (E D / 10)}{0.56065834919309210862625764267195 \times \ln \left(X_{C \text {-Eart }}\right)}$ & (140) \\
\hline$e=\ln \left(\frac{2 g_{0 \text {-Old }}}{\left(e^{X_{C-\text {-earn oLD }}}-1\right) \times 10^{10} \times p}\right)$ & (141) \\
\hline$e=10^{\frac{\log \left(\frac{T \mathrm{H}_{\text {OW }}+1}{100}+1\right)}{X_{C \text {-earthoLD }}}}$ & (142) \\
\hline$e=\ln \left(\frac{2 g_{0}}{T H \times 10^{8} \times p}\right)$ & (143) \\
\hline$e=10 \frac{\log \left[\frac{[\text { parsec in miles }}{0^{13}}\right]}{x_{C-\text { taranh }}}$ & (144) \\
\hline$e=\ln \left(\frac{2 g_{0}}{\text { Distance }_{\text {Lightw } \sim 1 \text { year }} \times 10^{-3} \times p}\right)$ & (145) \\
\hline 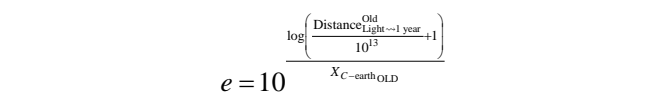 & (146) \\
\hline 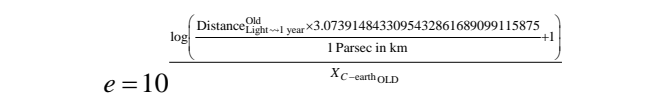 & (147) \\
\hline 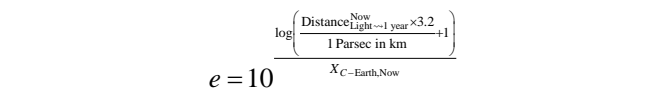 & (148) \\
\hline$e=\ln \left(\frac{g_{0 \text {-old }} \times \mathrm{SLCER}}{\mathrm{ER}_{\text {earth,Old }}}\right)$ & (149) \\
\hline$e=\ln \left(\frac{g_{0-\text { Now }} \times \text { SLCER }}{\text { ER }_{\text {earth,Now }}}\right)$ & (150) \\
\hline$\left.e=A W_{\text {C.C.F.P }} \times X_{C-\text { Earh }}=\left[4+\left(\left(\frac{V_{\text {C.C.F.P-Now }}}{\text { C.C.F.P. }}\right)-3\right)\right] \times X_{\text {UV }}\right)$ Earth & (151) \\
\hline 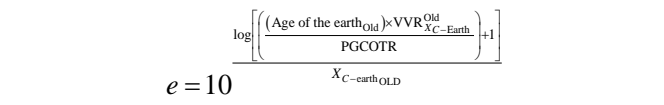 & (152) \\
\hline 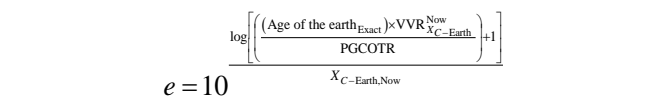 & (153) \\
\hline
\end{tabular}


Table 8. Continued

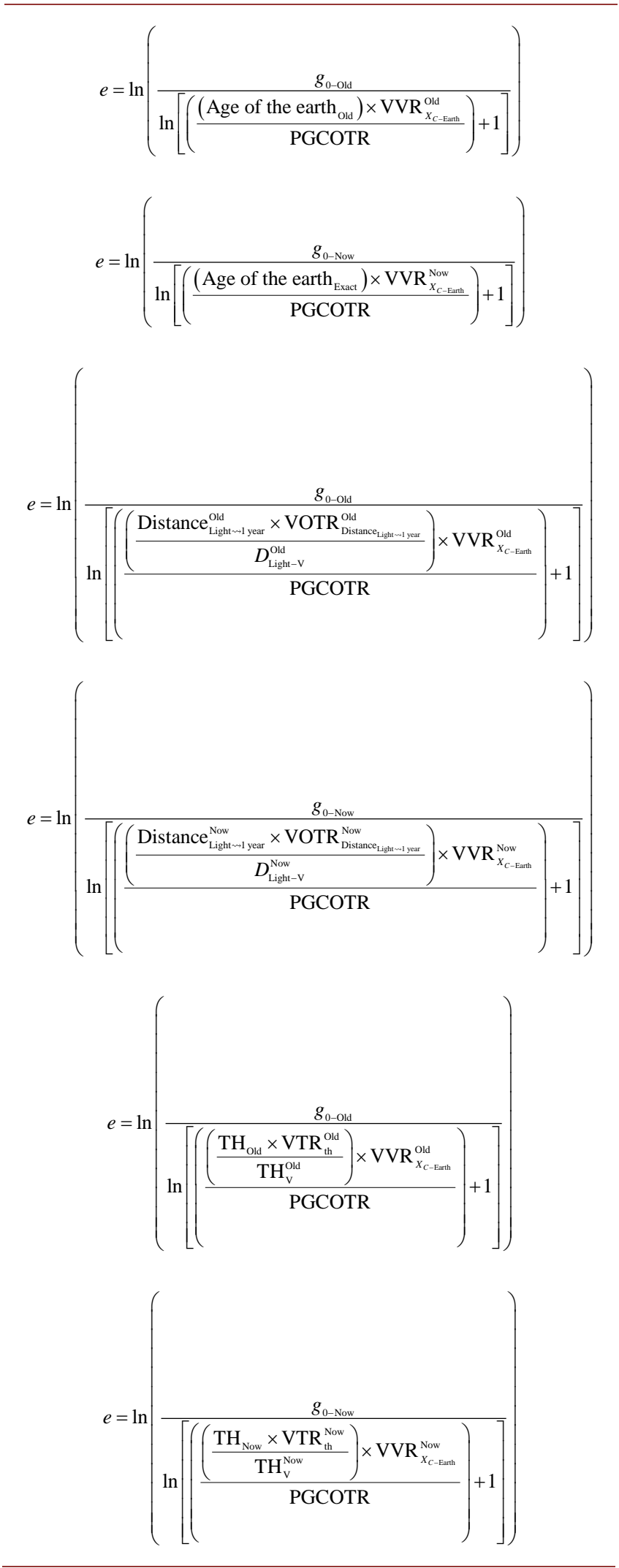

(154) 
Table 8. Continued

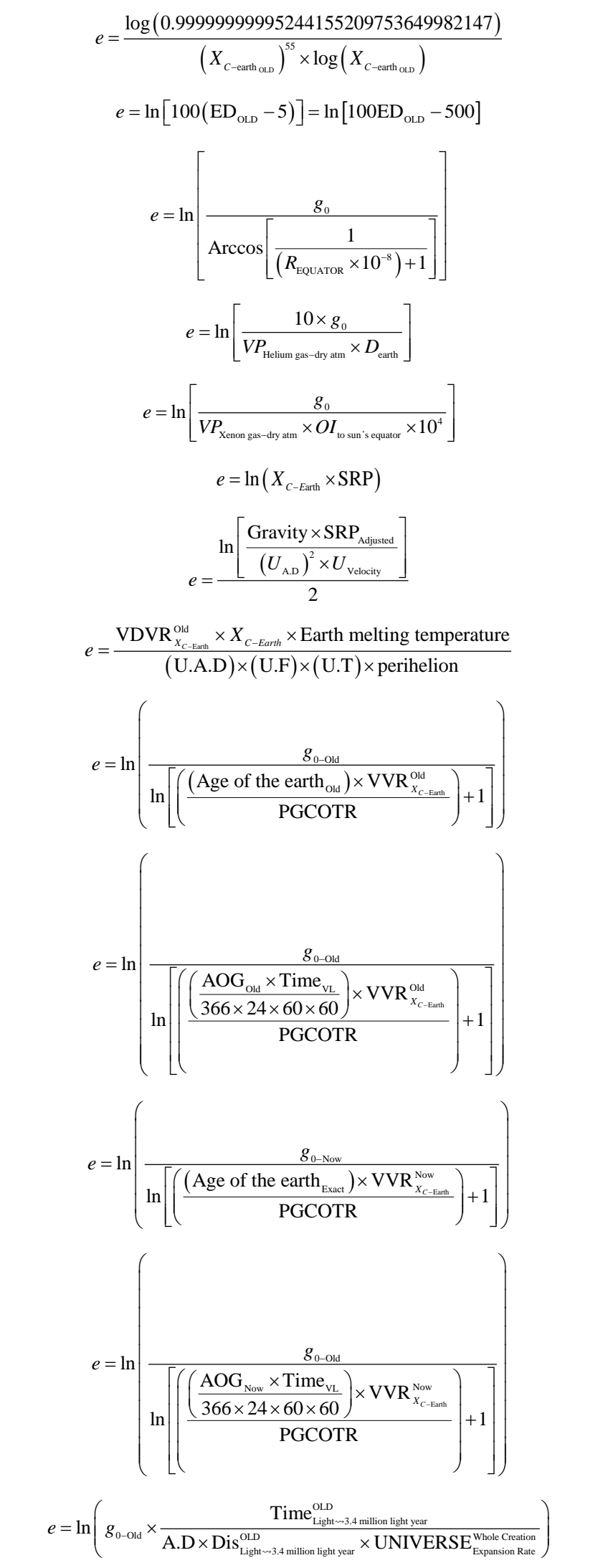


Table 8. Continued

$$
\begin{aligned}
& e=\ln \left(g_{0-\text { Now }} \times \frac{\text { Time }_{\text {Lightw3.335 million light year }}^{\text {Now }}}{\text { A.D } \times \operatorname{Dis}_{\text {Lightw.3.3.35 million light year }}^{\text {Now }} \times \operatorname{UNIVERSE}_{\text {Expansion Rate }}^{\text {begining point }}}\right) \\
& e=\ln \left(\frac{g_{0 \text {-Old }}}{\mathrm{AOE}_{\text {Old }} \times p}\right)=\ln \left(\frac{g_{0 \text {-old }}}{\mathrm{AOE}_{\text {Old }} \times \text { D.C } \times(\mathrm{MEARS})}\right) \\
& e=\ln \left(\frac{g_{0-\text { Now }}}{A O E_{\text {Exact }} \times p}\right)=\ln \left(\frac{g_{0-\text { Now }}}{\mathrm{AOE}_{\text {Exact }} \times \text { D.C } \times(\text { MEARS })}\right)
\end{aligned}
$$

\subsubsection{What are the Other Meanings of the Gravity and Mass of a Body? And How Can Unified Fundamental Forces of Nature in to One Unified Theory?}

Briefly, gravity is the cohesion between particles of the universe due to interactions between all forces to make stabilizing situation that effect on $0.06598803584531253707679018759685 \mathrm{Ggr}$ in 1 square meter of every parts of earth or everything near the earth. Therefore, follow relation is feasible based on Equations (18), (19), (21) and (22):

$$
\begin{aligned}
F & =m a=m g=m \frac{X_{C-\text { earth }}}{\text { A.D }}=\frac{M \times G \times E}{R_{\text {Earth }}^{2}}=\frac{R^{\prime} \times m \times G \times E}{R_{\text {Earth }}^{2}} \\
& =m \times \frac{G \times E}{R^{\prime \prime}}=m \times \frac{G \times E D \times V_{\text {Earth }}}{R^{\prime \prime}}
\end{aligned}
$$

According to the derived relationship between Gravitational Constant and speed of light by Zbiral deduces that light's speed would actually be a "dependent" universal constant of nature to $G$ and thus not a "genuine" constant of nature as shown as below [Adopted from [77]]:

$$
\frac{G}{C^{2}}=U_{\text {Cons. }}=7.4256484500928808373807285710012 \times 10^{28}
$$

Then, the gravitational constant can be written as:

$$
G=C^{2} \times U_{\text {Cons. }}=\frac{1}{\varepsilon_{0} \times \mu_{0}} \times U_{\text {Cons. }}
$$

Hence, from the Equations (1), (176) and (178), infer that:

$$
M=\frac{F \times R_{\text {Earth }}^{2}}{G \times E_{\text {Earth }}}=\frac{m \times g \times R_{\text {Earth }}^{2}}{G \times E_{\text {Earth }}}=\frac{m \times R_{\text {Earth }}^{2} \times X_{C-\text { earth }}}{\text { A.D } \times G \times E_{\text {Earth }}}
$$

By replacing the Equation (176) in the Equation (178) we have:

$$
F=M \times \frac{C^{2} \times U_{\text {Cons. }} \times E_{\text {Earth }}}{R_{\text {Earth }}^{2}}=M \times \frac{U_{\text {Cons. }} \times E D \times V_{\text {Earth }}}{\varepsilon_{0} \times \mu_{0} \times R_{\text {Earth }}^{2}}
$$

Therefore, strength limit based on speed of light, earth's mass, density, volume, area density and electrical and magnetic field constant is defined as bellows which shows how reach to a closer unified expressions of fundamental forces of nature:

$$
\begin{aligned}
X_{C-\text { Earth }} & =\frac{M}{m} \times \frac{C^{2} \times U_{\text {Cons. }} \times E D \times V_{\text {Earth }}}{R_{\text {Earth }}^{2}} \times \text { A.D } \\
& =R_{\text {Earth-Dimensionless }} \times \frac{U_{\text {Cons. }} \times E D \times V_{\text {Earth }}}{\varepsilon_{0} \times \mu_{0} \times R_{\text {Earth }}^{2}} \times \text { A.D }
\end{aligned}
$$


Above contents used to introduce a new illustrative definition for Mass. Mass is equal to the force involve on matter based on area density of earth with respect to its strength limit and the stabilized contraction of speed of light with the electrical and magnetic fields conditions which can be written directly as:

$$
m=\frac{F \times \text { A.D }}{X_{C-\text { earth }}}=\frac{F \times \text { A.D } \times C^{2} \times \varepsilon_{0} \times \mu_{0}}{X_{C-\text { earth }}}
$$

where, $U_{\text {Cons. }}$ is the ratio of $G$ to $C^{2}$ in $\mathrm{m} / \mathrm{kg}, \boldsymbol{\varepsilon}_{\mathbf{0}}$ is the electrical field constant, $\mu_{0}$ is the magnetic field constant, $\boldsymbol{C}$ is the speed of light and $\boldsymbol{R}_{\text {Earth-Dimensionless }}$ is the radius value of earth without unit (dimensionless).

\subsubsection{Featuring Exact Newtonian Constant of Gravitation}

As a historical review after newton calculation of gravitational constant in 16 century Cavendisch in 1798 inspired by the works of Coulomb in 1784 and 1785 (1784 Coulomb's report on his experiments on the torsion pendulum and 1785 Coulomb's work on the electrostatic force between two charges law determined using a pendulum) for the charges measures $G$ with a torsion pendulum. Eotovos in 1922 introduces his experiment on the equivalence of the inertial and gravitational mass. In 1982 Luther and Towler from National Institute of Standards and Technology (NIST) measure the G value equal to $6.67248(43) \times 10^{-11} \mathrm{~m}^{3}$ $\mathrm{kg}^{-1} \mathrm{~s}^{-2}$ through an improved data analysis of fiber torsion balance and dynamic mode. Fiber torsion balance method with dynamic mode used by Karagioz and Izmailov in 1996 from Tribotech Research and Development Company (TR\&D) to estimate G equal to $6.6729(5) \times 10^{-11} \mathrm{~m}^{3} \mathrm{~kg}^{-1} \mathrm{~s}^{-2}$ also Bagley and Luther in 1997 from Los Alamos National Laboratory (LANL), used it to evaluate G equal to $6.67398(70) \times 10^{-11} \mathrm{~m}^{3} \mathrm{~kg}^{-1} \mathrm{~s}^{-2}$. Gundlash and Merkowitz from University of Washington (UWash) in 2000 and 2002 presents a value of G with an improved experiment of fiber torsion balance method and dynamic compensation equal to $6.674255(92) \times 10^{-11} \mathrm{~m}^{3} \mathrm{~kg}^{-1} \mathrm{~s}^{-2}$. Quinn et al. from international Bureau of Weights and measures (BIPM) in 2001 calculates $G$ via strip torsion balance method by compensation mode and static deflection equal to $6.67559(27) \times 10^{-5}$. Kleinevoß and Kleinvoß et al. in 2002 from University of Wuppertal (UWup), applied suspended body method with displacement mode to compute $G$ equal to $6.67422(98) \times 10^{-11} \mathrm{~m}^{3} \mathrm{~kg}^{-1} \mathrm{~s}^{-2}$. Armstrong and Fitzgerald in 2003 from Measurement Standards Laboratory (MSL) through strip torsion balance method by compensation mode, assess $\mathrm{G}$ equal to $6.67387(27) \times 10^{-11} \mathrm{~m}^{3} \mathrm{~kg}^{-1} \mathrm{~s}^{-2}$. Hu et al. from Huazhong University of Science and Technology (HUST), in 2005 utilized fiber torsion balance method via dynamic mode to compute $G$ equal to $6.67228(87) \times 10^{-11}$. In 2006 Schlamminger et al. from University of Zurich (UZur) appraises $G$ by stationary body, weight change equal to $6.67425(12) \times$ $10^{-11} \mathrm{~m}^{3} \mathrm{~kg}^{-1} \mathrm{~s}^{-2}$. Luo et al. in 2009 and Tu et al. in 2010 from Huazhong University of Science and Technology (HUST) employ fiber torsion balance method through dynamic mode to rate the $G$ equal to $6.67349(18) \times 10^{-11} \mathrm{~m}^{3} \mathrm{~kg}^{-1} \mathrm{~s}^{-2}$. Parks and Faller in 2010 from University of Colorado and National Institute of 
Standards and Technology measure G via Suspended body method (displacement) equal to $6.67234(14) \times 10^{-11} \mathrm{~m}^{3} \mathrm{~kg}^{-1} \mathrm{~s}^{-2}$ [Adopted from [51]].

Accordingly, the measures of the Newtonian Gravitational factor are still based on method of torsion balance. The more recent methods are only smart improvements of the original one. They rely upon the use of the symmetries of the apparatus and synchronous detection.

But at the present the exact value of $G$ is demonstrates precisely through the strength limit, earth's gravity, density, volume, radius and mass also area density of earth's roles in accordance to Equation (23) as:

$$
\begin{aligned}
G & =\frac{X_{C-\text { Earth }} \times R^{\prime \prime}}{E D \times V_{\text {Earth }} \times \mathrm{A} . \mathrm{D}}=\frac{X_{C-\text { Earth }} \times \frac{R_{\text {Earth }}^{2}}{R^{\prime}}}{E D \times V_{\text {Earth }} \times \mathrm{A} . \mathrm{D}}=\frac{g \times \frac{R_{\text {Earth }}^{2}}{R^{\prime}}}{E D \times V_{\text {Earth }}}=\frac{g \times R_{\text {Earth }}^{\prime}}{E D \times V_{\text {Earth }}} \\
& =6.67384 \times 10^{-11} \mathrm{~m}^{3} \cdot \mathrm{kg}^{-1} \cdot \mathrm{s}^{-2}
\end{aligned}
$$

\subsubsection{How Can Compute Age of the Earth through Weil-Petersson Volumes, Symplectic Geometry, Intersection Theory and Moduli Spaces of Bordered Riemann Surfaces?}

Specifications of variations distance value range of earth's strength limit in 10 billion year according to old standard gravity, symplectic geometry, Riemann surfaces and their moduli spaces are determinant factors in appearance and accretion the age of the earth meanings. Age of the earth according to old standard gravity connected to the underlying theory structure of Weil-Petersson volumes, symplectic geometry, intersection theory, Riemann surfaces and their moduli spaces based on Equations (15), (107) and (110) defined as:

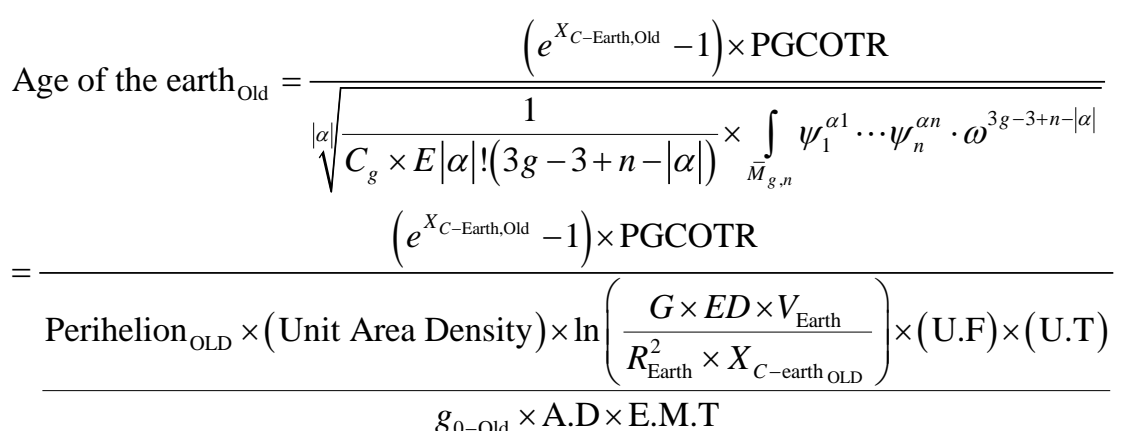

Strength limit through age of the earth shows covered bridges between several physical and mathematical systems as fundamental connection ways of Riemann surfaces and their moduli spaces and theoretical physics with hyperbolic geometry, complex analysis, topology, and dynamical systems as an important result.

\subsubsection{Introducing Other Descriptions of Area Density (Surface Density) According to Strength Limit and Specifications of Universe}

The area density ( $\mathrm{AD}$ or $\mathrm{SD}$ ) is a main quantity for the absorption of radiation. When studying bodies falling through air, area density is important because resistance depends on area, and gravitational force is dependent on mass. The Body mass index is in terms of area density [78]. Earth's strength limit has direct proportion to standard gravity. In variation status three cases made as follows: 1) 
Area density will be constant even if strength limit and standard gravity changed, 2) Area density will increase if strength limit and standard gravity changed, 3) Area density will decreases if strength limit and standard gravity changed. Above definitions of strength limit in old and present forms shows that area density in mentioned equations has a constant proportion. New various definitions of surface density are derived from Equations (2), (3), (14), (17), (19), (23), (34), (35), (37), (38), (83), (90), (99), (102), (107), (108), (112)-(115), (118), (121) and (128)-(130).

Equations (166) and (167) shows fiddling difference of area density value in calculation through old and present values of strength limit of earth as:

Surface density in accordance to present strength limit of earth = 0.06598803584531253707679018759687

Surface density in accordance to old strength limit of earth = $0.0659880358453125370767901875968 \underline{4}$

\section{Defining Perceptible Concepts}

Below perceptible meanings defined based on mentioned subjects:

A) Simplify ability of fundamental laws: Some complicated mathematical equations can illustrate by simple relations with have simple mathematical actions and special numbers such as: $\pi$, Napier's number, $e^{\mathrm{x}},\left(\mathrm{e}^{\mathrm{e}}\right)^{\mathrm{x}}$ and Golden section numbers.

B) Instability procedure law (I.P.L):

B1) Un-stabilization, rupturing and decomposing process of all things (structures, components and etc.) in existence (earth, planets, and universe) are like as descriptions in part 2.1.

And from the other side:

B2) Stabilization, conjoining and composing process of all things (structures, components and etc.) in existence (earth, planets, and universe) begun when their strength limit parameter increases continuously from $\left[\left(1-X_{c-\text { things }}\right) \times \mathbf{1 0 0}\right] \%$, to [(1-G.S.Ns number $) \times 100] \%$. Therefore after critical stages raise to $50 \%$. Besides strength limit parameter aggrandize to [G.S.Ns $\times 100] \%$ ([Golden section number $\times 100] \%),\left[\left(\left(\% X_{c-\text { things }}+\%\right.\right.\right.$ G.S.Ns $\left.\left.) / 2\right) \times \mathbf{1 0 0}\right] \%$ and $\left[X_{c \text {-things }} \times\right.$ $100] \%$ and secure adheres begin. Consequently, full secure complete composing and combining time and procedures occurs during and after that strength limit parameter heighten to $\left[\left(\left(1-X_{\underline{c}-\text { things }}\right)+\right.\right.$ G.S.Ns $\left.) \times \mathbf{1 0 0}\right] \%$ yet.

C) M.S.A's Napier (or Euler) limits: Determinant parameters such as $e, e^{x}$, $\left(e^{e}\right)^{x}$, and admixtures mathematical structures of " $\mathrm{e}$ " plays an importance role in existence (universe and world) cognition.

D) Cognitions by anthropic principles: Various aspects of the universe first can be understood using the anthropic principles and features of human, nature, earth and existence's components and their philosophy too.

E) Strength limit: Every structure and everything have strength limit parameter in various fields of science. This factor play vital role in every sciences. 
F) Principle Fundamental Pivot Numbers (PFPN): Numbers like strength limit and e and their changes are pivot numbers which shows the equivalency in the entire world and universe's components continuity in existence.

\section{Comprehensible General and Philosophical Approaches}

Perceptible general and philosophical approaches based on this study and discovering of this important sciences' parameter are as follows:

I) Existence is set of universe(s) and every contained thing(s) of them. All of the existence parts such as universe like earth have unity, correlation, continuity and dependency thereupon these specialties can be used to discover sciences and solving the problems. Regularity and dependency in existence such as cosmos has importance in sciences cognition and one of the applicable approaches.

II) Existence composed of visible and invisible. Unidentified can be a part of identified. In fact unidentified can be discovers with deep look at identified. All of the things are in front of your eyes but your efforts and tries appears them.

III) Whatever more learns lead to infinite unknowing. There are more different reasons about this subject and can be use every one of them for prescribe more questions. However humans are in the beginning way of the identifying of unknowns that these mysteries and unknowns create lots of questions in every human's mind. Different ideas said in various scientific fields until now but lots of them remain a simple theory. In fact unknowing will redound to known.

IV) Existology, particular anthropology is a way to discovers mysteries and unknowns. Existence and non-existence are oneness. Existence has unity and all of the people create one unit named human. Human and earth are small sample of existence's infinites. Existence, that's all things are importance in it and continuum together, hence can be discover elaborations of existence with scrutinizer consider in human's features punctuality. Therefore, deep look at human and earth specifications can help to knowing unidentified parts of universes which cause to explorations in sciences.

V) Complexities have simplicities and simples have complicates, thus can describe complicate cases as simple forms and overhand. Complicate and combined sciences in fact can understand by simples. Existence is a set of complexities and simplicities which interact together. Human can explains all of the components of the existence to simple(s) or complicate(s) forms or can be describe every parts of existence with scientific laws such as simple mathematics and physics laws.

VI) Every number has vast philosophy behind their mask and world interpretable by numbers' sciences.

VII) A very small discrepancy in numbers leads to creates lots of great different in features. Differences in features lead to discoveries in sciences.

VIII) In apparently manner a parameter is appear one factor but this parameter may be a combination of different or infinite factors.

IX) Behavior of numbers demonstrates regularities of the known or un-known 
$\operatorname{world}(\mathrm{s})$.

$\mathrm{X})$ All things in universe have some sign and allegory in human, nature and earth.

XI) All of the existence having intelligent life (brio). Life is intelligence.

XII) Scientific events in existence are dependent together.

XIII) All sciences, their structures and constituent components meet increasingly together and have common requirements to operating usage. Methodical explores succeed by working closely of all scientific areas along together.

\section{Summary, Discussion and Conclusions}

Strength limit of earth in fact is strength of interactions between fundamental gravity particles and gravity pull in reaching to equivalency and stability. Strength limit factor of earth helps to discovering the unity model of mathematical and physical frame works of the world, also algorithm structure of gravity and interactional impacts of particles together in universe. Combination of gravity with all other balancer and regularizer forces makes equivalency of the entire universe. Discoveries do not to be sufficient to recognize all-purpose of the world without imperfections as yet. Extended precision approaches involve in earth's strength limit will help to find complicates relations between various scientific features of universe to show a unified expressions of all interactions with independent background but via dependency to all existence components.

Strength limit of earth (S.L.E) is a unified and non-unified factor. Strength limit of other planets will be calculated by strength limit of earth. Decompose or collapse time of solar, galaxies and planets will predict by their strength limit parameter and earth strength limit. Gravity indicates having existence attraction balance energy between forces, elements, energies and situations. Gravitational collapses of earth and other planets will predict by their strength limit factors. Gravity forces have been unified. All units correlate themselves. Sum of all forces are one. Infinites and non-infinites have unity that confined for stability. Universe has expanding and earth has twitching also sides of earth have reducing now.

Strength limit of earth according to old standard gravity (the earth's gravity after the complete formation of the earth) is equal to 0.6471216 (MPa or \%) from every 1 unit (MPa or \%) of any strength forces or set of all resisting forces. And strength of existence global gravitation according to present standard gravity (the gravity of earth from initial time of earth's creation, during creation till the complete formation moment of the earth) is equal to 67.275 (MPa or \%) from every 100 unit (MPa or \%) of any strength forces or set of all resisting forces.

When earth created wholly in approximately 4.56 billion years, earth density is nearly equal to $5.5153 \mathrm{~kg} / \mathrm{m}^{3}$ which more than density value in 4.7304 billion years ago in the beginning time of the earth's creation. This shows that mass of the earth as well as density increased from beginning point of earth's creation 
process till full whole creation time. Then after whole creation time density and mass of the earth decreased by time passing.

The local or area impact velocity of gravity in the gravitational interactions field(s) distinguishing via the unit velocity of gravitational particles based on unit mass, time and force scales.

Humans' body conditions and specifications such as total number of cells in human body changed with variation in universe characteristics during their life time like as the universe expansion rate and age of the earth.

Differently Euler's number is a pivot number which its peculiarities dependent to fundamental particles' specifications.

The diversity between computed age of the galaxy in 2 determinant era in accordance to old and present resistive elasticity limit ascertain the exact origin, nature, time and characteristics of great climate, continental and structural changes for earth in the past, present and future.

There is an $180,190,186.3958982254670832584968$ years span which existed from the beginning point of the earth's creation process to the whole creation time. The 569,806,615.028565204501029363349 years difference between calculated ages of the galaxies shows $389,616,428.6326669790339461048522$ years interval after whole creation of the earth to stabilize in the galaxy system or approximately 389.62 million years interval between initial to whole creation point in completion procedures. These demonstrates

3.1622510993836013120810500861754 setting ages (eras) exist from the starting time of the earth's creation to whole creation and then to set in the galaxy system.

Any changes in speed of light variations and distance that light travel during one year cause to variety in time frame work of the earth and universe.

Present total number of seconds for a defined year, age of the earth, earth's orbital rotation features, earth density, principle great structural revolutionary mutations occurrence span, thickness of homosphere, distance that light travel during one year, particles' specifications moreover strength limit relates together (Equations (24)-(27), (131)-(134)). Each of them correlates with many other involved factors in universe and earth. If one parameter changed then others catch to varieties in cosmos continuously to make a global stabilized situation.

The relevancies of total number of seconds which exist in one year and distance that light travel during that same year appears any variation in speed of light phenomena also the conditions of electrical and magnetic fields which relates to light velocity. If total number of seconds which set for earth at the beginning point of its creation is as same as the whole creation time therefore the light velocity in that year face with higher amounts of value.

The definition of Euler's number based on earth's features like strength limit, gravity, mass, volume and density of earth and etc. assays and eventuates that universe and earth undergo 18 cycles of structural changing. Napier's limit notably is derived from itself amount in 18 cycles of series and parallel equation.

Strength limit which included gravity accounted well earth melting tempera- 
ture, strength limit of earth, Napier number, unit area density and other earth's features in determining the precession of the perihelion of earth via an alternatively generalized approach that seems a success for the ordinary Newtonian laws of gravitation.

Strength limit of earth display surface density role in precise determination of instant sidereal day, perihelion and earth melting temperature through earth's characteristic variations. Strength limit also area density of earth shows values of earth's obliquity to orbit and earth's sidereal day increased at earth's full whole creation time rather than beginning point of earth's creation process.

Specify distinctly the accurate total number of seconds for any defined time applied to adjusting units such as the exact astronomical unit and assigning the precise earth distance from the sun and the accurate age of the earth.

According to the Equation (182) mass is equal to the force acting on matter in stabilized interactional gravitational, electrical and magnetic fields in accordance to earth's area density and strength limit roles in the light field. These can help to discovery of an integrated procedure for unifying fundamental forces of nature in to one structure.

Universe's expansion rate according to age of the earth since start time of earth's creation view point is defined 67.275 and the rate after 4.7304 billion years is 73.03652362904 .

Many scientists and researches centers suggested various amounts for the cosmic speed measurements but they did not know about the origins were made them also the era that these measures appertain too. Age, resistive elasticity factor and gravity of earth appear the universe's expansion rate alterations during earth and universe life time.

Gravitational particles' decay parameter variety illustrates existence of unknown principal fundamental elements and particles. These peculiarities use to discover other fundamental equalizer forces of universe and earth.

The relation between total number of seconds and ELTGP demonstrates that exact life time of gravitational particles according to unit of time for the whole creation procedures of earth is lower than its value in the beginning time of the earth's creation process.

Suggested equations demonstrates extremely small amount variation of area density value in universe's life time passing based on old and present values of earth's strength limit.

No scientists can compute scrutinizing value of constant of gravitation and its probable variations pending universe life time since Newton calculation of gravitational constant at sixteen century as yet. Strength limit through earth's gravity, density, volume, radius and mass also area density proving the accurate value of $\mathrm{G}$ which researchers struggle with during centuries.

As a considerable result strength limit and age of the earth display unknown fundamental correlations between several physical and mathematical disciplines with hyperbolic geometry, Riemann surfaces and their moduli spaces, complex analysis, topology, and dynamical systems. 
Strength limit provide assessment of earth's features in different ages.

Strength limit factor for earth has the greatest superlative phenomenal usages in discovering of correlations between all sciences and all parts of cosmos.

Moreover dimensional constant and variable aspects of strength limit of earth, it's a dimensionless physical constant (instantly or for a period of time) from the other side which can be calculates its value at any moment and also will use to derive other dimensionless physical constants, their numbers, measures, and values. Scientists will enable to response to the dimensionless physical constants problem through these traits.

Philosophy of strength limit factor of earth shows that complications of every scientific subject's structures have a real simple potential truth(s) which concealed in them.

Strength limit delineate frameworks which:

- Contemplate all properties of cosmos.

- Unification of all involved parts of the cosmos and their interactions.

- One fundamental parameter introduces all other quintessential factors and constants also uncover their basilar roles.

- Can use as a connector of different corrected theories together.

- Construct comprehensive scientific models such as physical and mathematical frameworks too.

- Does not make divergences at all.

- Inclusion of gravity in any sciential systems and subsystems.

- Indicate and gauge any perturbations and invariances in involved scientific factors, systems and frameworks,

- It can contemplate super-symmetries and higher or infinite dimensions.

\section{Significances of This Study for Future of Scientific Researches}

Some others subjects which can survey scrutinizingly based on strength limit in separate research articles are:

Predicting future of the earth and universe according to instant changes; Determine the time of earth decomposing; Determining strength of fundamental forces such as gravity, electromagnetism and weak and strong forces; Earth magnetic fields mysteries (Why earth has magnetic fields? What are all the reasons of its existence and extensions?); Finding luminary similar to earth if exist; Description of time concept in existence creation, in universe creation and in creation of earth and other things that visible or invisible; Finding and adjusting errors in physics theories such as: Einstein's theories; Developing of units concept; Developing of dimensions concept; Helping in earthquake predicting (Predicting earthquakes by strength limit of earth before occurrences); The unification like the unification of the universe and its forces; What does the origin of the unification mean?; How important is it to understand the stability of DNA?; What is the genius rebound (mutation) threshold?; What is the genius variation 
threshold?; Is our universe unique, or are there many universes?; How can detected accurately gravitational waves experimentally?; Accurate studying on amending and reforming of gene (human genome); Finding decisive curing ways of immedicable disease like HIV; Help to better knowing of black holes; Describe the process of DNA replication in terms of subatomic properties.; Are there particles smallest than presented known ones? Are there particles which their velocities faster than light? Are there basis particles that make structures and more efficacious than higgs? Is there particle that make genitures and ruptures of universe structures? When were the first stars formed, and what were they like? Why are the galaxies distributed in clumps and filaments? ...

Strength limit number of earth has many practical results, applications and advantages excessively such as it will be solve most important physics problems which some of them here they are: $\left(\mathrm{Q}_{1}\right)$. Are all the (measurable) dimensionless parameters that characterize the physical universe calculable in principle or are some merely determined by historical or quantum mechanical accident and incalculable? $\left(\mathrm{Q}_{2}\right)$. How can quantum gravity help explain the origin of the universe? $\left(\mathrm{Q}_{3}\right)$. What is the lifetime of the proton and how do we understand it? $\left(Q_{4}\right)$. Is nature super symmetric, and if so, how is super symmetry broken? $\left(Q_{5}\right)$. Why does the universe appear to have one time and three space dimensions? $\left(\mathrm{Q}_{6}\right)$. Why does the cosmological constant have the value that it has, is it zero and is it really constant? $\left(\mathrm{Q}_{7}\right)$. What are the fundamental degrees of freedom of $\mathrm{M}$-theory (the theory whose low-energy limits is eleven-dimensional super gravity and which subsumes the five consistent superstring theories) and does the theory describe nature (String theory and M-theory dualities)? $\left(\mathrm{Q}_{8}\right)$. What physics explains the enormous disparity between the gravitational scale and the typical mass scale of the elementary particles? $\left(\mathrm{Q}_{9}\right)$. Can we quantitatively understand quark and gluon confinement in quantum chromo dynamics and the existence of a mass gap? $\left(\mathrm{Q}_{10}\right)$. How can we merge quantum theory and general relativity to create a quantum theory of gravity? How can we test this theory? $\left(\mathrm{Q}_{11}\right)$. Will a complete physical model of the world help us to understand ultimate reality? Can we understand ultimate reality at all through science? $\left(\mathrm{Q}_{12}\right)$. Is the proton really stable, or does it eventually decay? $\left(\mathrm{Q}_{13}\right)$. Why think each generation of particles has precisely this structure: two leptons and two quarks? Does it true? $\left(\mathrm{Q}_{14}\right)$. Thermonuclear fusion $\left(\mathrm{Q}_{15}\right)$. Turbulence $\left(\mathrm{Q}_{16}\right)$. High temperature superconductivity $\left(\mathrm{Q}_{17}\right)$. Bonus problem; metallic hydrogen and exotic matter $\left(\mathrm{Q}_{18}\right)$. Cosmological constant inflation...

Moreover the various parts discussed in above, strength limit factor can response to the follow problems: How the exact frames work of the universe?; How the physics of the universe?; Determining age of the humankind life on earth; Determining age of the life on earth; Determine the neutrino velocity and the characteristics of neutrino's mass; Determining life time of particles such as proton; Safety factor calculation of a slope on earth and other planets; Description of errors in rock engineering and rock mechanics theories; Prediction age 
and decompose time of the stars, planets and galaxies even in far and extreme points and etc.; How was our universe born? What happened when the universe first emerged just after the very instant of creation? How was the beginning of the universe?; what is the size of the whole universe? Does a multiverse exist?; Comprehending changes and events that occur for earth in future.; Do the quarks or leptons have any substructure, or are they truly elementary particles? Is standard model of fundamental particles perfect?; How can we merge quantum theory and general relativity to create a quantum theory of gravity? How can we test this theory? Where is the center of the universe or world or existence? What's the age and life time of the earth's magnetic fields? What's the relation between strength limit parameter and overthrow of creatures in various ages? Is there any infinite energy in universe? What relevance exists between strength limit parameter and $\infty$ ? How fundamental particles change climate conditions and ecosystems? How clean areas that infected by atomic and nuclear actions with different methods such as fundamental and mater particles? How gravity effect on particles on ultra-microscopic scale?

Besides strength limit able to solve fundamental questions in various parts of general physics, quantum physics, cosmology, general relativity, quantum gravity, high-energy and particle physics, astronomy and astrophysics, nuclear physics, atomic, molecular and optical physics, condensed matter physics, plasma physics, biophysics such as: Can quantum mechanics and general relativity be realized as a fully consistent theory (perhaps as a quantum field theory)? [79]; Is space-time fundamentally continuous or discrete? Would a consistent theory involve a force mediated by a hypothetical graviton, or be a product of a discrete structure of space-time itself (as in loop quantum gravity)? Are there deviations from the predictions of general relativity at very small or very large scales or in other extreme circumstances that flow from a quantum gravity theory? [1]; What gives rise to the Standard Model of particle physics? Why do particle masses and coupling constants have the values that we measure? Why are there three generations of particles?; Why is there more matter than antimatter in the universe? Where does Dark Matter fit into the model? Is it even a new particle? [33]; Is string theory, superstring theory, or M-theory, or some other variant on this theme, a step on the road to a "theory of everything", or just a blind alley? [80]; How to unified classical and quantum mechanics, classical and quantum field theory, general and special relativity, string theory, loop quantum gravity and all beneficial parts of existed scientific theories to make a basis framework for knowing all components of the entire universe?

Above explanations are few advantages of this study for the future of the scientific researches. Likewise, strength limit be able to response to complicated unsolved science's problems.

\section{Interests}

The author declares that he (Author) is the owner of all interests of this article 
forever.

\section{Descriptions of Author}

Mohammad Salehi Alashti (M.S.A.) proposed the idea for the project and planned it. Author conceived, designed, performed, interpreted and organized this research study and wrote the paper. M.S.A. performed all measurements, analyzed the data, compiled and discussed the results and implications also reviewed the manuscript. Author read and approved the final manuscript. Author provided the original work is properly credited.

\section{Funding Statement}

No support used for implementation of this research study.

\section{Competing Interest Statement}

The author declares that there has been not any conflict of interest.

\section{License Statement}

This article published under the terms of the Author's private license.

\section{Funding statement}

No financial support used for implementation of this research study.

\section{References}

[1] Grillo, G. Loop Quantum Gravity. https://en.wikipedia.org/wiki/Loop_quantum_gravity

[2] Sakurai, J.J. and Napolitano, J. (2010) Modern Quantum Mechanics. 2nd Edition, Pearson, 68.

[3] Novello, M. and Bergliaffa, S.E. (2003) Cosmology and Gravitation: Xth Brazilian School of Cosmology and Gravitation; 25th Anniversary (1977-2002), Mangaratiba, Rio de Janeiro, Brazil, Springer Science \& Business Media, 95.

[4] Superstring. https://en.wikipedia.org/wiki/Superstring

[5] Classical Unified Field Theories. https://en.wikipedia.org/wiki/Classical_unified_field_theories

[6] 't Hooft, G. and Veltman, M. (1974) One Loop Divergencies in the Theory of Gravitation. Annales de P Institut Henri Poincaré, 20, 69.

[7] Veltman Martinus, J.G. (1975) Quantum Theory of Gravitation-Course 5. In: Balian, R. and Zinn-Justin, J., Eds., Methods in Field Theory-Les Houches Session XXVIII Summer School in Theoretical Physics, 77, North Holland Publishing Company, Netherlands, Oxford, New York, and World Scientific Publishing, Singapore.

[8] Goroff, M.H. and Sagnotti, A. (1985) Quantum Gravity at Two Loops. Physics Letters $B, 160,81-86$. https://doi.org/10.1016/0370-2693(85)91470-4

[9] Donoghue, J.F. (1995) Introduction to the Effective Field Theory Description of Gravity. In: Cornet, F. and Herrero Marie, J., Eds., Proceedings of the Advanced School on Effective Theories, Almunecar, Spain, 1 Jan. 1997 Effective Theories, World Scientific Publishing Co Pte Ltd., Singapore. 
[10] Weinberg, S. (1996) The Quantum Theory of Fields 2: Modern Applications. Cambridge University Press, Cambridge. https://doi.org/10.1017/CBO9781139644174

[11] Hamber, H.W. (2009) Quantum Gravitation-The Feynman Path Integral Approach. Springer Publishing.

[12] Zee, A. (2010) Quantum Field Theory in a Nutshell. 2nd Edition, Princeton University Press, 172.

[13] Penrose, R. (2007) The Road to Reality: A Complete Guide to the Laws of the Universe. Vintage, 1017.

[14] Dirac, P.A.M. (1930) A Theory of Electrons and Protons. Proceedings of the Royal Society A, 126, 360. https://doi.org/10.1098/rspa.1930.0013

[15] Anderson, C.D. (1933) The Positive Electron. Physical Review, 43, 491-494. https://doi.org/10.1103/PhysRev.43.491

[16] Special Relativity. https://en.wikipedia.org/wiki/Special_relativity

[17] Messiah, A. (1981) Quantum Mechanics 2. North-Holland Publishing Company, 875.

[18] Nicolai, H., Peeters, K. and Zamaklar, M. (2005) Loop Quantum Gravity: An Outside View. Classical and Quantum Gravity, 22, R193-R247. https://doi.org/10.1088/0264-9381/22/19/R01

[19] Goswami, J., Pankaj, S. and Singh, P. (2006) Quantum Evaporation of a Naked Singularity. Physical Review Letters, 96, 31302. https://doi.org/10.1103/PhysRevLett.96.031302

[20] Rovelli, C. (2000) Notes for a Brief History of Quantum Gravity.

[21] Green, M.B., Schwarz, J.H. and Witten, E. (1987) Superstring Theory. Volume 1: Introduction. Cambridge University Press, Cambridge.

[22] Ibanez, L.E. (2000) The Second String (Phenomenology) Revolution. Classical \& Quantum Gravity, 17, 1117-1128. https://doi.org/10.1088/0264-9381/17/5/321

[23] History of String Theory. https://en.wikipedia.org/wiki/History_of_string_theory

[24] Douglas, M.R. (2003) The Statistics of String/M Theory Vacua. Journal of High Energy Physics, 0305, 46. https://doi.org/10.1088/1126-6708/2003/05/046

[25] Ashok, S. and Douglas, M. (2004) Counting Flux Vacua. Journal of High Energy Physics, 0401, 60. https://doi.org/10.1088/1126-6708/2004/01/060

[26] Rickles (2014) A Brief History of String Theory: From Dual Models to M-Theory. The Frontiers Collection, Springer, 230-236

[27] van Nieuwenhuizen, P. (1981) Supergravity. Physics Reports, 68, 189-398. https://doi.org/10.1016/0370-1573(81)90157-5

[28] Woit, P. (2011) Implications of Initial LHC Searches for Supersymmetry.

[29] Cassel, S., Ghilencea, D.M., Kraml, S., Lessa, A. and Ross, G.G. (2011) Fine-Tuning Implications for Complementary Dark Matter and LHC SUSY Searches. Journal of High Energy Physics (JHEP), 5, 120. https://doi.org/10.1007/JHEP05(2011)120

[30] Falkowski, A. (Jester) (2011) What LHC Tells About SUSY. http://resonaances.blogspot.com/

[31] Tapper, A. (2010) Early SUSY Searches at the LHC. Imperial College, London.

[32] Jha, A. (2013) One Year on from the Higgs Boson Find, Has Physics Hit the Buffers? The Guardian. Photograph: Harold Cunningham/Getty Images. GMG, London.

[33] Standard Model. https://en.wikipedia.org/wiki/Standard_Model 
[34] CERN (2010) Particle Chameleon Caught in the Act of Changing.

[35] Blumhofer, A. and Hutter, M. (1997) Family Structure from Periodic Solutions of an Improved Gap Equation. Nuclear Physics B, 484, 80-96. https://doi.org/10.1016/S0550-3213(96)00644-X

[36] M-Theory. https://en.wikipedia.org/wiki/M-theory

[37] Giudice, G.F. (2013) Naturalness after LHC8. PoS EPS. HEP, 163. http://arxiv.org/pdf/1307.7879.pdf

[38] Composite Higgs Models. https://en.wikipedia.org/wiki/Composite_Higgs_models

[39] Newton's Laws of Motion. https://en.wikipedia.org/wiki/Newton\%27s_laws_of_motion

[40] Lubliner, J. (2008) Plasticity Theory. Revised Edition, Dover Publications.

[41] Salehi Alashti, M. (2015) Accurate Age of the Earth Calculation by a New Fundamental Parameter. Open Access Library Journal, 2, e1296. https://doi.org/10.4236/oalib.1101296

[42] United States Department of Commerce, National Institute of Standards and Technology (2008) The International System of Units (SI), Vol 330. In: Taylor, B.N. and Thompson, A., Eds., NIST Special Publication, National Institute of Standards and Technology, Gaithersburg, 52. http://nvlpubs.nist.gov/nistpubs/Legacy/SP/nistspecialpublication330e2008.pdf

[43] Cohen, B. (2002) The Investigation of Difficult Things: Essays on Newton and the History of the Exact Sciences in Honour of D.T. Whiteside. In: Harman, P.M. and Shapiro, A.E., Eds., The Investigation of Difficult Things, Cambridge University Press, Cambridge, 353.

[44] Plastino, A.R. and Muzzio, J.C. (1992) On the Use and Abuse of Newton's Second Law for Variable Mass Problems. Celestial Mechanics and Dynamical Astronomy, 53, 227-232. Kluwer Academic Publishers, Netherlands. https://doi.org/10.1007/BF00052611

[45] Newton, I. (1999) The Principia: Mathematical Principles of Natural Philosophy. Translated by Bernard, I., Cohen and Whitman, A. Preceded by a Guide to Newton's Principia by I. Bernard Cohen Assisted by Julia Budenz. University of California Press, California, Proposition 75, Theorem 35, 956.

[46] Ehlers, J. (1973) Survey of General Relativity Theory. In: Werner, I., Ed., Relativity, Astrophysics and Cosmology, Academic Publishing Company, Springer, Netherlands, Dordrecht-Holland, 16. https://doi.org/10.1007/978-94-010-2639-0_1

[47] Kenyon, I.R. (1990) General Relativity. Oxford University Press, Oxford, 244 p.

[48] Mohr, P.J., Taylor, B.N. and Newell, D.B. (2012) CODATA Recommended Values of the Fundamental Physical Constants: 2010. Reviews of Modern Physics, 84, 1527.

http://physics.nist.gov/cuu/Constants/Preprints/lsa2010.pdf

http://physics.nist.gov/cuu/Constants/index.html https://doi.org/10.1103/RevModPhys.84.1527

[49] Weinberg, S. (1972) Gravitation and Cosmology: Principles and Applications of the General Theory of Relativity. John Wiley \& Sons Inc., New York, 688 p. http://www.wiley.com/WileyCDA/WileyTitle/productCd-0471925675.html

[50] Groten, E. (2012) The Geodesist's Handbook 2012 Part 5: General Information; Geodetic Standards and Conventions. In: International Association of Geodesy (IAG) Office and IAG Communication and Outreach Branch (COB), Ed., Springer Journal of Geodesy, 86, 962-963. 
http://link.springer.com/content/pdf/10.1007\%2Fs00190-012-0584-1.pdf

[51] Mohr, P.J., Taylor Barry, N. and Newell, D.B. (2012) CODATA Recommended Values of the Fundamental Physical Constants: 2010 (2012 Adjustment of 2010 CODATA Values). Journal of Physical and Chemical Reference Data, 41, 043109. https://arxiv.org/abs/1203.5425 https://doi.org/10.1063/1.4724320

[52] Mohr, P.J., Taylor, B.N. and Newell, D.B. (2015) CODATA Recommended Values of the Fundamental Physical Constants: 2014. National Institute of Standards and Technology, Gaithersburg. https://doi.org/10.6028/NIST.SP.961r2015

[53] Clabon Walter, A. (2000) Allen's Astrophysical Quantities, Chapter 2: General Constants and Units Part 3: General Astronomical Constants. In: Cox, A.N., Ed., 4th Edition, AIP Press, Springer-Verlag, New York, 12.

[54] Williams, D.R. (2004) Earth Fact Sheet. NASA Goddard Space Flight Center, Greenbelt, MD 20771. http://nssdc.gsfc.nasa.gov/planetary/factsheet/earthfact.html

[55] US Geological Survey (1997) The Age of the Earth. Branch of Isotope Geology, United States Department of the Interior, United States Geological Survey, Menlo Park, California. http://pubs.usgs.gov/gip/geotime/age.html http://geomaps.wr.usgs.gov/parks/gtime/ageofearth.html

[56] Bianconi, E., Piovesan, A., Facchin, F., Beraudi, A., Casadei, R., Frabetti, F., Vitale, L., Pelleri Maria, Ch., Tassani, S., Perez-Amodio, F., Piva, S., Strippoli, P. and Canaider, S. (2013) An Estimation of the Number of Cells in the Human Body. Annals of Human Biology, 40, 463-471.

http://www.tandfonline.com/doi/full/10.3109/03014460.2013.807878

https://doi.org/10.3109/03014460.2013.807878

[57] Wikipedia Articles on Atmosphere (2016). http://en.wikipedia.org/wiki/Atmosphere_of_Earth https://en.wikipedia.org/wiki/Layers_of_the_atmosphere

[58] James, B. and Scott, T. (2008) Galactic Dynamics. 2nd Edition, Princeton University Press, Princeton and Oxford, Princeton.

[59] Groten, E. (2000) Parameters of Common Relevance of Astronomy. Geodesy and Geodynamics Journal of Geodesy, 74, 134-140. http://link.springer.com/article/10.1007/s00190-000-0134-0

[60] Luzum, B., Capitaine, N., Fienga, A., Folkner, W., Fukushima, T., Hilton, J., Hohenkerk, C., Krasinsky, G., Petit, G., Pitjeva, E., Soffel, M. and Patrick, W. (2011) The IAU 2009 System of Astronomical Constants: The Report of The IAU Working Group on Numerical Standards for Fundamental Astronomy. Celestial Mechanics and Dynamical Astronomy, 110, 293-304.

http://link.springer.com/article/10.1007\%2Fs10569-011-9352-4

https://doi.org/10.1007/s10569-011-9352-4

[61] Williams, D.R. (2006) Planetary Fact Sheets. http://nssdc.gsfc.nasa.gov/planetary/factsheet/

[62] Lewars, E.G. (2008) Modelling Marvels. Computational Anticipation of Novel Molecules. Springer, Springer Science and Business Media B.V., The Netherlands, 70-71.

[63] Weiss Ray, F. (1971) Solubility of Helium and Neon in Water and Seawater. Journal of Chemical \& Engineering Data, 16, 235-241. http://pubs.acs.org/doi/abs/10.1021/je60049a019?journalCode=jceaax https://doi.org/10.1021/je60049a019

[64] Merriam-Webster (1991) The Merriam-Webster New Book of Word Histories. Mer- 
riam-Webster Incorporated, Springfield, Massachusetts, 513.

[65] (2000) CRC Handbook of Chemistry and Physics, Internet Version 2005 Edition, CRC Press LLC, N. W. Corporate, Boca Raton. http://www.hbcpnetbase.com

[66] Wikipedia Article on Earth (2016) http://en.wikipedia.org/wiki/Earth

[67] Dalrymple, G.B. (1991 \& 1994) The Age of the Earth. Stanford University Press, California.

[68] Dalrymple, G.B. (2001) The Age of the Earth in the Twentieth Century: A Problem (Mostly) Solved. Geological Society of London Special Publications, 190, 205-221. http://sp.lyellcollection.org/content/190/1/205.abstract

[69] Newman, W.L. (2007) Age of the Earth. Publications Services, USGS, U.S. Government Printing Office, Washington DC.

https://pubs.er.usgs.gov/publication/70039222

[70] Stassen, C. (1997) The Age of the Earth. TalkOrigins Archive; Exploring the Creation and Evolution Controversy, 1.

http://www.talkorigins.org/faqs/faq-age-of-earth.html

[71] Staff (2007) Useful Constants. International Earth Rotation and Reference Systems Service.

[72] Mirzakhani, M. (2007) Weil-Petersson Volumes and Intersection Theory on the Moduli Space of Curves. Journal of the American Mathematical Society, 20, 1-23. http://math.stanford.edu/ mmirzakh/Papers/WPI.pdf https://doi.org/10.1090/S0894-0347-06-00526-1

[73] Bizouard, C. (2014) Useful Constants. International Earth Rotation and Reference Systems Service. http://hpiers.obspm.fr/eop-c/models/constants.html

[74] Zeilik, M. and Gregory, S.A. (1998) Introductory Astronomy \& Astrophysics. 4th edn, Saunders College Publishing, 56.

[75] Clara, M. (2016) Cosmic Speed Measurement Suggests Dark Energy Mystery. Scientific American, Space and Physics Editor's Note.

http://www.scientificamerican.com/article/cosmic-speed-measurement-suggests-da rk-energy-mystery

[76] Wall, M. (2016) The Universe May Be Expanding Faster Than Astronomers Thought. Scientific American.

http://www.scientificamerican.com/article/the-universe-may-be-expanding-faster-t han-astronomers-thought/ http://www.SPACE.com

[77] Zbiral, G. (2012) Does Gravitation Have an Influence on Electromagnetism? Journal of Modern Physics, 3, 1223-1230. http://www.SciRP.org/journal/jmp

[78] Visconti, G. (2001) Fundamentals of Physics and Chemistry of the Atmosphere. Springer, Berlin, 470. http://www.springer.com/us/book/9783662045404

[79] Sokal, A. (1996) Don't Pull the String Yet on Superstring Theory. New York Times. https://query.nytimes.com/gst/fullpage.html?res=9D0DE7DB1639F931A15754C0A 960958260

[80] Theory of Everything (2017)

https://en.wikipedia.org/wiki/Theory_of_everything 